\title{
MAP7 Prevents Axonal Branch Retraction by Creating a Stable Microtubule Boundary to Rescue Polymerization
}

\author{
(DStephen R. Tymanskyj and ${ }^{-L e}$ Ma \\ Department of Neuroscience, Jefferson Synaptic Biology Center, Vickie and Jack Farber Institute for Neuroscience, Sydney Kimmel Medical College, \\ Thomas Jefferson University, Philadelphia, Pennsylvania 19107
}

Complex neural circuits are built from axonal branches that allow each neuron to connect with multiple targets. During development, maturation of nascent branches depends on stabilization of newly assembled or transported microtubules, which are thought to be regulated by microtubule-associated proteins (MAPs). However, because many known MAPs inhibit branch formation, it is not clear which MAP is responsible for regulating microtubule stability during branch development. Here, we show that MAP7, a less-well understood MAP that is localized to branch junctions, provides a key molecular mechanism to regulate microtubule stability during branch formation. In developing rodent sensory neurons of mixed sex, MAP7 is required for branch maturation mainly by preventing branch retraction. This function is mediated by the ability of MAP7 to control microtubule stability, as microtubules are more stable at branch junctions where MAP7 is localized. Consistently, nascent branches depleted of MAP7 have decreased stable microtubules but increased dynamic microtubules. Moreover, MAP7 binds to the acetylated and stable region of individual microtubules and avoids the dynamic plus end, thereby creating a boundary that prevents microtubule depolymerization and rescues microtubule polymerization. This unique binding property, which is not observed for other MAPs, can prevent branch retraction caused by laser-induced severing or nocodazole-induced microtubule depolymerization. Together, our study identifies a novel molecular mechanism mediated by MAP7 to regulate microtubule stability and strengthen branches at different stages of axonal branch morphogenesis.

Key words: axonal branch development; MAP7; microtubule; sensory neurons

Significance Statement

Development and maintenance of axonal branches rely on microtubule stability, but the underlying molecular mechanisms are not fully understood. Here, we show that MAP7, a unique protein that interacts with both microtubules and the motor protein kinesin-1, plays a key role at branch junctions. MAP7 stabilizes microtubules in nascent branches and prevents branch retraction during branch maturation or after laser-induced injury. MAP7 also binds to the acetylated region of microtubules to prevent depolymerization and rescue polymerization. This unique binding property supports a novel mechanism mediated by MAP7 to cooperate with other MAPs and control microtubule stability during axonal branch development. This mechanism could also impact microtubule regulation in branch regeneration after nerve injury.

\section{Introduction}

Establishing the complex neural connectivity depends on the generation of elaborate axonal branches that allow a single neuron to connect with multiple synaptic targets (O'Leary et al.,

Received April 1, 2019; revised July 16, 2019; accepted July 31, 2019.

Author contributions: S.R.T. and L.M. designed research; S.R.T. performed research; S.R.T. and L.M. analyzed data; S.R.T. and L.M. wrote the paper.

This work was supported by an NIH R01 Grant to L.M. (NS062047) and grants from the Farber Discovery Fund and the Jefferson Synaptic Biology Center of the Thomas Jefferson University. We thank Peter Bass for sharing plasmid DNAs for Tau-EGFP and EB3-mcherry, members of the Dalva laboratory for sharing rat tissues, Bridget Curran for helpful discussion, and Ben Yang and Angelo Lepore for comments on the manuscript.

The authors declare no competing financial interests.

Correspondence should be addressed to Le Ma at le.ma@jefferson.edu.

https://doi.org/10.1523/JNEUROSCI.0775-19.2019

Copyright $\odot 2019$ the authors
1990; Acebes and Ferrús, 2000; Gibson and Ma, 2011; Rockland, 2019). Formation of these branches is achieved through various signaling pathways, all of which ultimately converge on the regulation of key cytoskeleton components, actin and microtubule (Gallo, 2011; Lewis et al., 2013; Kalil and Dent, 2014; Kapitein and Hoogenraad, 2015). Actin-based filopodia initiate branch formation along the axon; microtubules then invade the filopodia via polymerization or transport to generate nascent branches that continue to grow and become mature branches (Gallo, 2011; Kalil and Dent, 2014). However, nascent branches can retract if microtubules are not stabilized inside them (Gallo, 2011; Lewis et al., 2013; Kalil and Dent, 2014). The ability to control branch growth and retraction is thus critical to establishing stereotypic and plastic synaptic connections, as demonstrated by recent 
studies of the formation of layer-specific collateral branches in cortical pyramidal neurons (Hand et al., 2015) and synapse elimination in the neuromuscular junction (Brill et al., 2016).

During branch development, it is thought that microtubules are stabilized by microtubule-associated proteins (MAPs) in nascent branches (Gallo, 2011; Kalil and Dent, 2014). Several MAPs, including tau, MAP1B, and doublecortin, which bind or crosslink microtubules, have been proposed to stabilize microtubules to promote branch maturation (Kalil and Dent, 2014). However, these MAPs are often present uniformly along the axon or at the axonal terminal (Dotti et al., 1987; Tint et al., 2009; Tymanskyj et al., 2010); and expression perturbation studies show that these MAPs suppress branch formation (Koizumi et al., 2006; Yu et al., 2008; Bilimoria et al., 2010; Tymanskyj et al., 2012; Ketschek et al., 2015). Therefore, it is not clear which specific MAP is responsible for stabilizing microtubules during nascent branch maturation.

In this study, we investigated MAP7, a MAP that can promote branch formation, especially branch maturation of rodent dorsal root ganglion (DRG) sensory neurons (Tymanskyj et al., 2017, 2018). MAP7 is enriched at branch junctions, making it an ideal candidate to stabilize microtubules in nascent branches. MAP7 has two microtubule binding domains that contribute to its high microtubule binding affinity (Tymanskyj et al., 2018) and competes against tau for microtubule binding in vitro (Monroy et al., 2018). MAP7 shows strong recruitment of kinesin-1 to microtubules via a carboxyl terminal domain (Sung et al., 2008; Metzger et al., 2012; Barlan et al., 2013; Tymanskyj et al., 2018) and can influence kinesin-1 based organelle transport in neurons (Tymanskyj et al., 2018). Although the kinesin interaction allows MAP7 to regulate organelle transport, the microtubule binding property of MAP7 that is essential for recruiting kinesin to microtubules has largely been overlooked. Additionally, how MAP7 affects microtubule stability critical to branch growth remains to be studied.

Using a gene-trap mouse mutant (Komada et al., 2000) that produces Map7-null neurons, we show that MAP7 is required for branch maturation by preventing branch retraction. We show that the MAP7-rich regions in axons and branch junctions contain more stable microtubules and MAP7 deletion or overexpression has opposing effects on microtubule stability in these axonal regions. We also show that MAP7 is needed to increase microtubule stability in nascent branches. These functions are achieved by MAP7 binding to specific regions along microtubules that generates a boundary to prevent microtubule depolymerization and rescue polymerization, a property specifically seen with MAP7 but not the axonal MAP, tau. This unique binding property supports MAP7 to prevent retraction of laser-severed mature branches and influence branch retraction caused by nocodazoleinduced microtubule depolymerization. Our combined data thus provide strong evidence to support a microtubule regulatory mechanism governed by MAP7 to regulate axonal branch development and maintenance.

\section{Materials and Methods}

DNA constructs, animals, and cell lines. EGFP expression constructs for MAP7 (FL and N) were described previously (Tymanskyj et al., 2017, 2018). 3R-tau-EGFP (Qiang et al., 2006) and EB3-mCherry (Qiang et al., 2010) were gifts from Dr. Peter Baas (Drexel University). Timed pregnant Sprague-Dawley rats were obtained from Charles River Laboratories and used in accordance with the Guidelines for the Care and Use of Laboratory Animals of the National Institutes of Health and the approved IACUC protocol (\#01560) of the Thomas Jefferson University. The
$\mathrm{Map7}^{-1-}$ mouse line (Komada et al., 2000) was obtained from The Jackson Laboratory (JAX stock \#004153) and maintained in accordance with the approved IACUC protocol (\#01558, \#01559). Vaginal plug dates were designated as E0.5 for mice and E0 for rats. COS-7 cells (originally from the stock of the Tessier-Lavigne laboratory) were grown in DMEM with $10 \%$ fetal bovine serum (Invitrogen).

Culture of primary DRG neurons and COS cells. Primary rat DRG neuronal cultures were performed as described previously (Zhao et al., 2009). Briefly, DRGs were dissected out from E17 rat embryos of mixed sex, washed once in HBSS, and incubated at $37^{\circ} \mathrm{C}$ with $0.25 \%$ trypsin for 10-15 min. Trypsin-treated DRGs were resuspended in L15 medium plus $10 \%$ horse serum and then mechanically triturated with a firepolished glass pipette. Dissociated rat DRG neurons $\left(\sim 7.5 \times 10^{5}\right.$ cells $)$ were transfected with various MAP7 or tau constructs by Nucleofection (Lonza) using reagent $\mathrm{P} 3$ and the $\mathrm{CU}-133$ program. Neurons were then plated at $\sim 20,000$ cells on $18 \mathrm{~mm}$ glass coverslips coated with $10 \mu \mathrm{g} / \mathrm{ml}$ poly-D-lysine $/ 10 \mu \mathrm{g} / \mathrm{ml}$ laminin and cultured in F12 medium (with the $\mathrm{N} 3$ supplement, $40 \mathrm{~mm}$ glucose, and $25 \mathrm{ng} / \mathrm{ml} \mathrm{NGF}$ ). After $24 \mathrm{~h}$, cells were either fixed or subjected to live imaging as described in the following sections.

$M a p 7^{-1-}$ neurons were collected from E15.5 mouse embryos of mixed sex. DRGs with the same genotype were then pooled and processed for culture as described for rat neurons. Dissociated neurons were imaged live or grown for $24 \mathrm{~h}$ before fixation.

COS cells were transfected using a TransMax system (gift from Yun Yao, University of Southern California) with various DNA constructs and plated on glass coverslips or glass bottom dishes (MatTek; Tymanskyj et al., 2018). For microtubule polymerization experiments, EB3mCherry was cotransfected along with EGFP tagged constructs. After overnight culture, cells were fixed or subjected to live imaging described in the following sections.

Immunocytochemistry, imaging, and quantification. After overnight culture, neurons or COS cells were either fixed in 4\% PFA/PBS for morphology analysis or 4\%PFA/0.5\% glutaraldehyde in PBS for microtubule analysis. For the nocodazole treatment (Baas and Black, 1990), DRG neurons were treated either with $6.6 \mu \mathrm{M}$ nocodazole (NocF) or with DMSO control (CtrlF) in the medium for 15 min before fixation with methanol.

Cells were stained with the following primary antibodies: $\alpha$-tubulin (1:5000; Abcam; RRID:AB_2210057), $\alpha$-tubulin (DM1 $\alpha ; 1: 2000$, Sigma-Aldrich; RRID:AB_521686), C-terminal MAP7 (1:1000; GeneTex; RRID:AB_11170884), acetylated tubulin (1:1000; Sigma-Aldrich; RRID:AB_477585), tyrosinated tubulin (YL1/2, Bio-Rad; 1.2000; RRID: AB_325003) or neurofilament (RMO270, 1:1000; Sigma-Aldrich; RRID: AB_2315286). EGFP-tagged proteins in DRG neurons and COS cells were visualized directly by the fluorescence of the EGFP tag or by labeling with an EGFP antibody (Aves Labs; RRID:AB_10000240). For antibody labeling, cells were blocked in PBS plus 5\% goat serum and $0.1 \%$ Triton (PGT) for $1 \mathrm{~h}$ and then incubated with primary antibodies diluted in PGT for at least $1 \mathrm{~h}$ at room temperature or overnight at $4^{\circ} \mathrm{C}$. After washing, they were incubated with $\mathrm{Cy} 3$ and/or Cy5-labeled secondary antibodies (Jackson ImmunoResearch) diluted in PGT for $1 \mathrm{~h}$ at room temperature. Digital images were taken on a Yokogawa spinning disk confocal system using 488,560 , or $680 \mathrm{~nm}$ laser excitation with a $20 \times$ or $100 \times$ objective. Images are shown as 2 -dimensional projections with specific pseudo-colors based on the LUTs in Image J.

Based on digital confocal images, the following parameters were analyzed in ImageJ as previously described (Tymanskyj et al., 2017): number of branches per $100 \mu \mathrm{m}$ axon, main axon length, branch length, and number of primary neurites per neuron. Primary axons were counted as any processes $>20 \mu \mathrm{m}$ extending from the cell body. The longest of them, measured from the cell body to the growth cone, was considered as the primary axon. Branches $>10 \mu \mathrm{m}$ were traced, counted, and normalized to the primary axon length. They were divided into two groups based on their location along axons (terminal vs interstitial) as defined in the Results section. Branch numbers for each neuron were summed and normalized to the axon length of each individual neuron.

For fluorescence intensity measurements in DRGs, lines were drawn along the axon or branch region and gray values along the lines were acquired as indicated in the figure. For the MAP7 overexpression level, 
the fluorescent intensity of MAP7 antibody staining was compared between nontransfected and FL-EGFP-expressing neurons in axon and branch regions.

Live cell imaging. E15.5 mouse DRG neurons were dissociated and cultured in laminincoated glass bottom dishes. The dish was mounted onto a heated and humidified chamber (Okolab) equilibrated to $37^{\circ} \mathrm{C}$ with $5 \%$ $\mathrm{CO}_{2}$ on an inverted microscope (Axiovert 200, Zeiss). Both phase contrast and fluorescent images were acquired using a spinning disk confocal system (Yokogawa, W1) with an sCMOS camera (Zyla, Andor) or an EMCCD camera (Cascade 512, Photometrics). For branch dynamic analysis, images were taken using a $40 \times$ objective every $5 \mathrm{~min}$ for $10-18 \mathrm{~h}$. For filopodia analysis, images were taken using a $63 \times$ objective every 2 min for $30 \mathrm{~min}$. Image contrast was auto adjusted in ImageJ using $\mathrm{min} /$ max values.

In branch dynamic analysis, branch growth refers to branches that appeared and extended $>20 \mu \mathrm{m}$ over the length of imaging time (between 6 and $18 \mathrm{~h}$ ) and is represented by the number of growth events per hour. Branch retraction refers to the complete retraction of the newly formed branch back to the axon and is represented by the number of retraction events per hour. The length and the lifetime before retraction were also recorded for each branch. These parameters were analyzed in $>10 \mathrm{WT}$ or mutant neurons from $>3 \times$ experiments. They were divided into two groups based on the modes of branching (bifurcation and collateral branch formation) as described in the Results section.

EB3 imaging in live COS cells was performed $24 \mathrm{~h}$ after transfection. Images were captured on a spinning disk confocal system with an EMCCD camera at 2-4s time intervals for a total of $4 \mathrm{~min}$. Kymographs were generated using ImageJ. For EB3 analysis, the plusTipTracker (Applegate et al., 2011) was used to generate EB3 speeds and growth lengths. For the nocodazole treatment, cells were set up for live imaging of EB3, followed by perfusion with fresh media containing $15 \mu \mathrm{M}$ nocodazole. Cells were monitored until the majority of EB3 signals had been removed. For washout, fresh media without nocodazole was perfused while cells were imaged.

Analysis of branch response after laser-severing or nocodazole treatment. Rat DRG neurons were transfected with various DNA constructs, grown overnight, and then moved to the on-stage culture system (Okolab). Images were acquired using $488 \mathrm{~nm}$ laser or phase contrast on a spinning disk confocal system by an sCMOS camera (Zyla, Andor). Neurons were injured using a Micropoint (Andor) with the minimum power setting to achieve severing of the axon. Successful severing was identified as complete separation of the axon at the site of injury by phase contrast imaging. Initial images were acquired using a $63 \times$ objective every $1 \mathrm{~min}$ for 20 min, subsequently followed by imaging every $5 \mathrm{~min}$ for $1 \mathrm{~h}$. For nocodazole experiments, imaging was done the same way after rat or mouse neurons were treated with $15 \mu \mathrm{M}$ nocodazole.

In situ analysis. Cryosections (16-20 $\mu \mathrm{m}$ ) were processed following a standard procedure (Zhao et al., 2009) using DIG-labeled riboprobes. The Map7-specific RNA probes were subcloned into the pCRII-TOPO vector (Invitrogen) with the following primers: $\mathrm{N}$-terminal probe $(250$ bp): forward, ATCCCCCACCTGTGCTACG; reverse, CTTGTCCTCC significant. Scale bars, $100 \mu \mathrm{m}$.
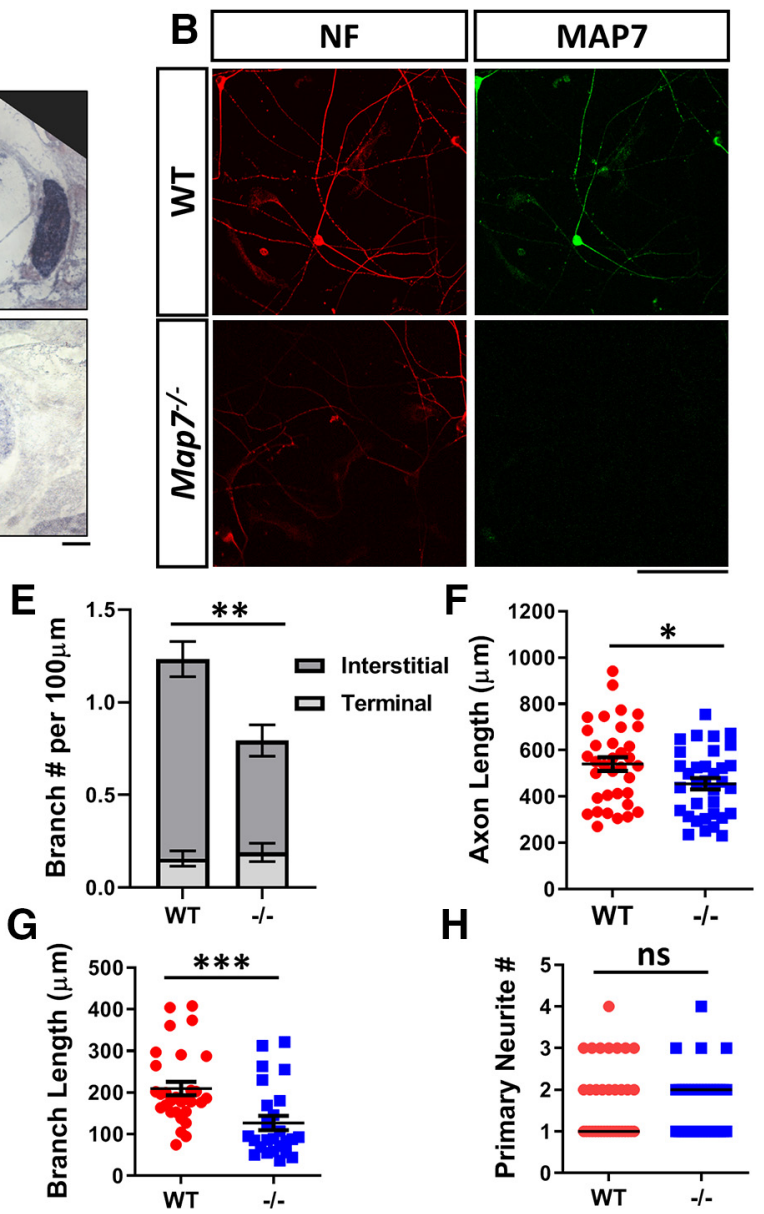

Figure 1. Loss of MAP7 decreases axon branching in DRG neurons. $\boldsymbol{A}$, In situ analysis of E15.5 mouse spinal cords from WT and $=30$ for WT; $n=25$ for $-/-$, Mann-Whitney test), and primary neurite number $(\boldsymbol{H} ; p=0.8894, n=35$ for WT, $n=39$ for - / - Mann-Whitney test). Data are reported as mean \pm SEM in $\boldsymbol{E}-\mathbf{G}$, and median in $\boldsymbol{H}^{*} p<0.05 ;{ }^{* *} p<0.01 ;{ }^{* * *} p<0.001$; ns, not

TCCAGCCTCTG; C-terminal probe (543 bp), forward: ACAGCAAT CAGAAGTGACCACAGAGAG; reverse, TGTCTGCACACCATCCACCT.

Experimental design and statistical analysis. All measurements are reported as mean \pm SEM, except that Figure $1 H$ is shown by the median. Statistical analysis was performed in Prism 8.0 software (GraphPad). Data were first tested for normality by the Kolmogorov-Smirnov. Normally distributed data were compared by unpaired two-tailed $t$ tests for two samples or one-way ANOVA with Tukey's post hoc analysis for three or more samples. Data that were not normally distributed were analyzed by the Mann-Whitney test for two samples or by the Kruskal-Wallis test for three or more samples. The statistical test, the sample size $(n)$, and the $p$ values are reported in the figure legend or the Results section. $P$ values $<0.05$ are considered significant and represented by asterisks.

\section{Results}

MAP7 is required for axonal branch maturation by preventing branch retraction

To understand the role of MAP7, we analyzed DRG neurons isolated from a gene-trap mouse line in culture. The mouse line was created by an insertion of a $\beta$-gal gene-trap cassette into intron 1 , which disrupts MAP7 at amino acid 23 and thus creates a null allele (Komada et al., 2000). Absence of MAP7 expression was confirmed by in 
A

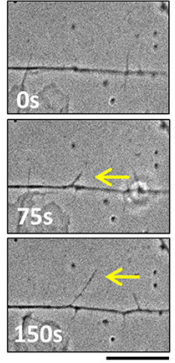

B

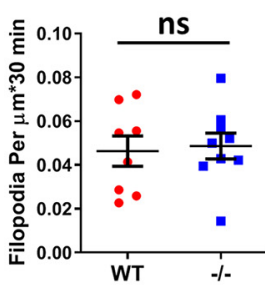

C

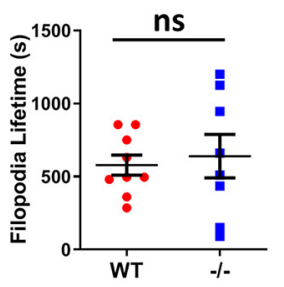

D

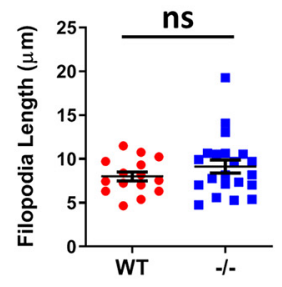

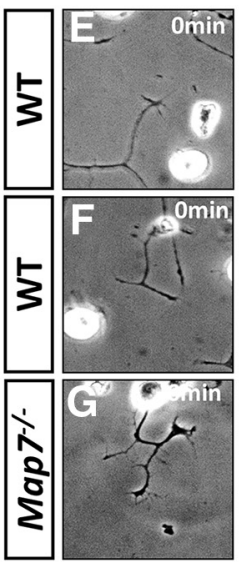

H

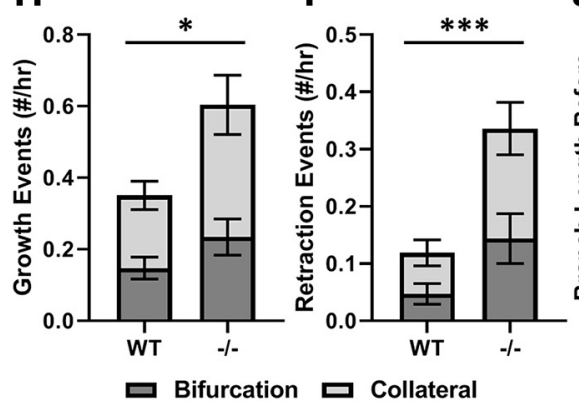

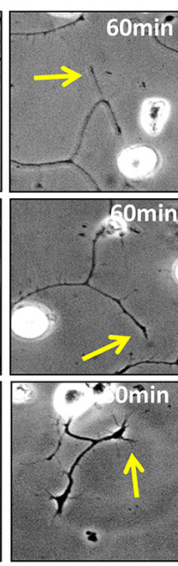

1

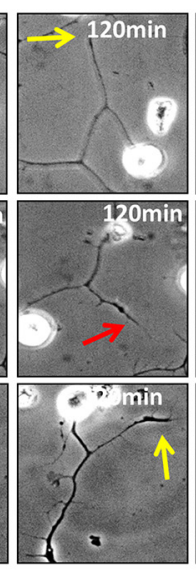

$\mathbf{J}$

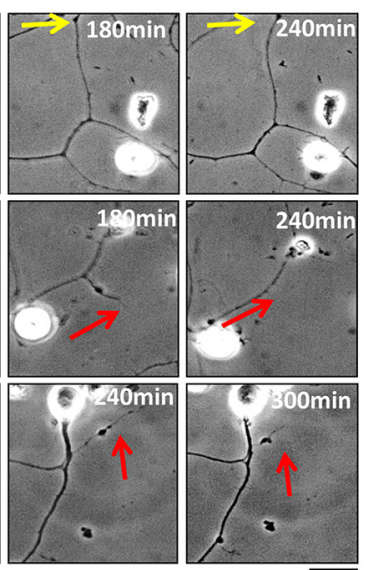

$\mathrm{K}$

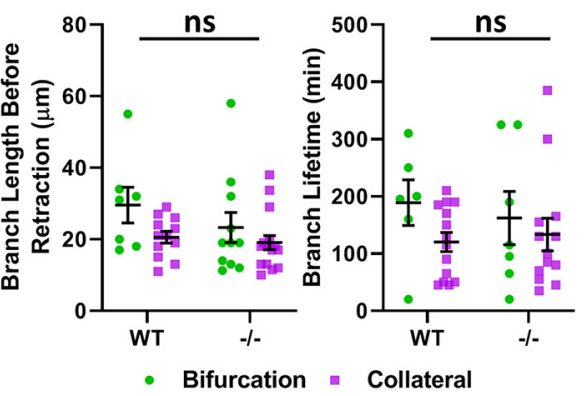

Figure 2. Loss of MAP7 increases axonal branch retraction in DRG neurons. $A$, Formation of filopodia (arrows) is shown by sequential phase contrast images of an axonal segment. $\boldsymbol{B}-\boldsymbol{D}$, Comparisons between WT and $\mathrm{Map}^{-1-}(-/-)$ neurons in filopodium formation frequency ( $\boldsymbol{B} ; p=0.8026, n=8$ for WT and $n=9$ for $-/-$, unpaired $t$ test), filopodium lifetime $(\boldsymbol{C} ; p=$ $0.7047, n=9$ for WT and $n=8$ for $-/$, , unpaired $t$ test), and filopodium length ( $\boldsymbol{D} ; p=0.2575, n=15$ for WT and $n=21$ for $-/-$, unpaired $t$ test). $\boldsymbol{E}-\boldsymbol{G}$, Examples of sequential phase contrast images (60 min apart) to show branch growth (yellow arrows) and branch retraction (red arrows) of WT $(\boldsymbol{E}, \boldsymbol{F})$ and $\mathrm{Map}^{-/-}(\boldsymbol{G})$ neurons. $\boldsymbol{H}-\boldsymbol{K}$, Comparison between WT and mutant $(-/-)$ neurons of branch growth $(\boldsymbol{H}$; total $p=0.0169$, bifurcation $p=0.1318$, collateral $p=0.0510 ; n=18$ cells for WT and $n=10$ cells for $-/$ - ; unpaired $t$ test), branch retraction $(I$; total $p=0.0157$, bifurcation $p=0.0244$, collateral $p=0.0204 ; n=18$ cells for WT and $n=10$ cells for $-/-$; Mann-Whitney test), branch length before retraction (J; total $p=0.1092, n=19$ for WT and $n=$ 27 for $-/-$; bifurcation, $p=0.2948, n=7$ for WT and $n=11$ for $-/-$; collateral, $p=0.2344, n=12$ for WT and $n=16$ for $-/-$; Mann-Whitney test), and branch lifetime before retraction $(K$; total $p=0.3267, n=20$ for WT and $n=20$ for $-/-$; bifurcation, $p=0.3590, n=6$ for WT and $n=7$ for $-/-$; for collateral, $p=0.5808, n=14$ for WT and $n=13$ for $-/-$; unpaired $t$ test). Data are reported as mean \pm SEM. ${ }^{*} p<0.05{ }^{* * *} p<0.001$; ns: not significant (only shown for the total in $\boldsymbol{H}-\boldsymbol{K}$ ). Scale bars: $20 \mu \mathrm{m}$.

situ analysis in spinal cord sections (Fig. $1 A$ ) and antibody staining of MAP7 and neurofilament (NF) in cultured DRG neurons (Fig. 1B) of wild-type (WT) and Map $7^{-1-}$ animals.

Consistent with previous studies (Tymanskyj et al., 2017), DRG neurons isolated from E15.5 WT mouse embryos had exuberant branches (Fig. 1C), producing $1.23 \pm 0.11$ total branches per $100 \mu \mathrm{m}$ axon in culture (Fig. $1 E$ ). However, DRG neurons from $M a p 7^{-1-}$ littermates displayed a 37\% decrease in the number of total branches per $100 \mu \mathrm{m}$ axon to $0.79 \pm 0.10$ (Fig. $1 D, E$ ). The reduction in branches was due to a $44 \%$ decrease in intersti- tial branches, defined as those extending from the proximal $90 \%$ of the axon, from $1.08 \pm 0.10$ branches in WT neurons to $0.60 \pm 0.08$ branches in $\mathrm{Map}^{-1-}$ neurons per $100 \mu \mathrm{m}$ axon. However, there were no significant differences in terminal branches, defined as those from distal $10 \%$ axons, between WT and $\mathrm{Map}^{-1-}$ neurons $(0.16 \pm 0.04$ vs $0.19 \pm 0.05$ branches per $100 \mu \mathrm{m}$; Fig. $1 E$ ). Loss of MAP7 did not affect the formation of axons, but the main axon produced by mutant neurons were shortened by $17 \%$ (WT: $540.2 \pm 29.3 \mu \mathrm{m}$ vs $\mathrm{Map7}^{-1-}$ : $445.8 \pm 24.7 \mu \mathrm{m}$; Fig. $1 F)$. Because the reduction in branch number is larger than that of axonal length, the impact on branch formation by the loss of MAP7 is not merely a secondary consequence of primary axonal growth. Additionally, the total branch length was reduced by $39 \%$ $(209.9 \pm 16.0 \mu \mathrm{m}$ in WT vs $127.0 \pm 17.1$ $\mu \mathrm{m}$ in $\mathrm{Map7}^{-1-}$; Fig. $1 G$ ) without affecting the neurite number per cell (Fig. $1 H$ ), further supporting a role of MAP7 in branch growth. These results demonstrate that MAP7 is required for axon growth, branch formation, and branch growth, consistent with the previous analysis by shRNA knockdown (Tymanskyj et al., 2017) and domain expression (Tymanskyj et al., 2018).

Our previous studies of precocious expression of MAP7 in DRG neurons suggested that MAP7 is required for branch maturation but not branch initiation (Tymanskyj et al., 2017). To test these different roles, we used live-cell imaging (Fig. 2A) to analyze filopodia formation, the initial step in branch development. WT neurons normally produce $\sim 0.046 \pm 0.007$ filopo$\mathrm{dia} / \mu \mathrm{m}$ every $30 \mathrm{~min}$ and $M a p 7^{-1-}$ neurons showed no significant difference $(0.048 \pm 0.006$ filopodia/ $\mu \mathrm{m} / 30 \mathrm{~min}$; Fig. $2 B)$. Further comparison of the lifetime and length of filopodia showed that neither was affected by the loss of MAP7 (Fig. 2C,D). These data demonstrate that MAP7 is not required for branch initiation and suggest its role in branch maturation.

To understand the precise role of MAP7 in branch maturation, we used livecell imaging to obtain more detailed analysis of nascent branch dynamics (Fig. 2E-G). Time-lapse movies (every $5 \mathrm{~min}$ for $10-18 \mathrm{~h}$ ) showed that nascent branches $>10 \mu \mathrm{m}$ often undergo growth and retraction from the axon (Fig. $2 E-G$, yellow and red arrows). We quantified the growth and retraction events and found that WT DRG neurons produced $0.35 \pm 0.05$ new nascent branches every hour (Fig. $2 \mathrm{H}$ ) with some of them retracting at a rate of $0.11 \pm 0.03$ event/h (Fig. $2 I$ )

Live-cell imaging also allows us to distinguish two modes of branching: bifurcation and collateral branch formation. Bifurca- 
A
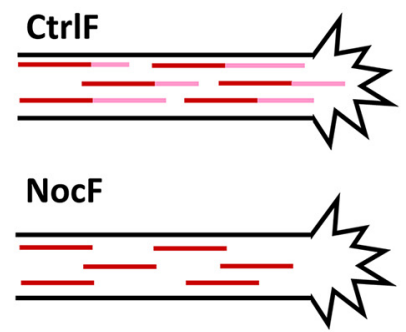

Stable MT

Dynamic MT

B

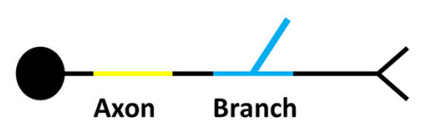

C

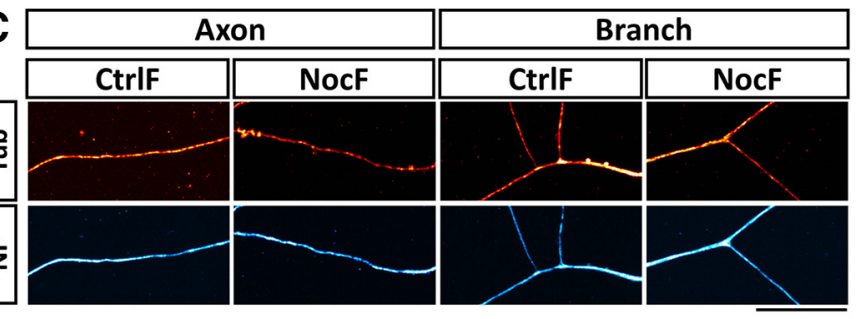

D

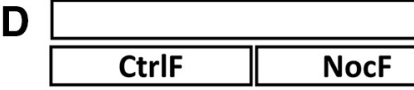

Axon

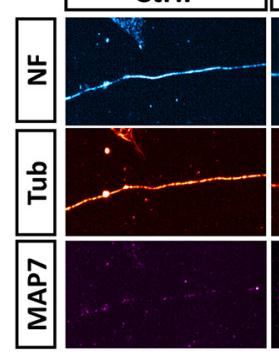

G
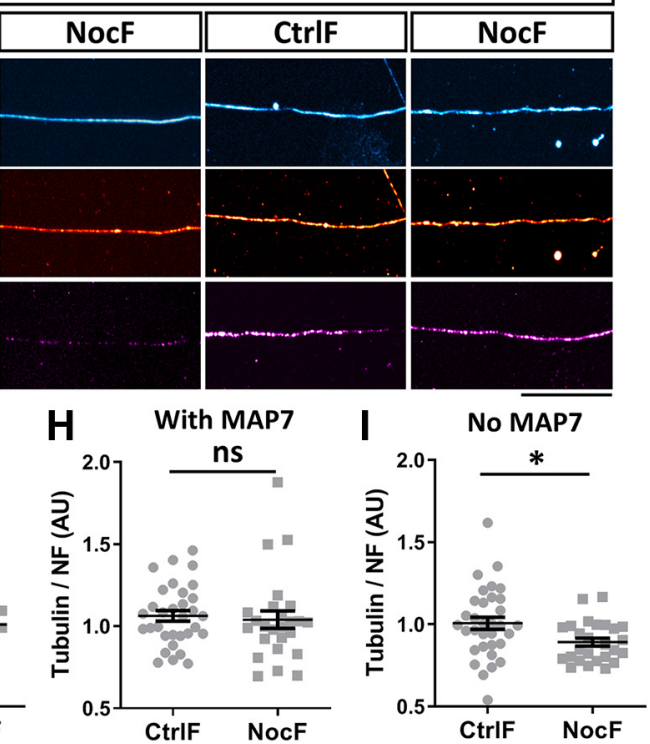

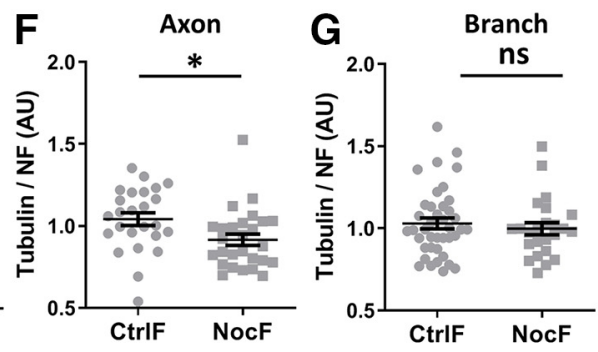

Figure 3. MAP7 maintains stable microtubules at branch regions. $A$, Schematic of the use of the CtrlF or NocF method in neurons. Treatment of neurons with nocodazole before fixation removes dynamic microtubules (pink) leaving behind only stable microtubules (red). $\boldsymbol{B}$, Schematic of different regions of the neuron analyzed. The axonal region is shown in yellow and the branch region in blue. C, Fluorescence intensity images of axon or branch regions labeled for tubulin (tub, red hot) and NF (cyan hot) after CtrlF or NocF. D, Fluorescence intensity of axonal regions with (right) or without (left) MAP7 (magenta hot) that are labeled for tubulin (tub, red hot) and NF (cyan hot) after CtrlF or NocF.E, Comparison of the neurofilament labeling after CtrlF or NocF ( $p=0.8670, n=$ 39 for CtrlF and $n=41$ for NocF, unpaired $t$ test). $F, G$, Quantification of the fluorescence signal ratio of tubulin over NF in axon regions $(\boldsymbol{F} ; p=0.019, n=25$ for $C$ trlF and $n=27$ for NocF, unpaired $t$ test) or branch regions $(\boldsymbol{G} ; p=0.529, n=40 \mathrm{for}(\mathrm{trlF}$ and $n=24 \mathrm{for}$ NocF, unpaired $t$ test). $\boldsymbol{H}, \boldsymbol{I}$, Quantification of the fluorescence signal ratio of tubulin/NF in axonal regions with $(\boldsymbol{H} ; p=0.4015$, $n=32$ for CtrlF and $n=25$ for NocF, Mann-Whitney test) or without MAP7 (l; $p=0.0175, n=33$ for CtrlF and $n=26$ for NocF, unpaired $t$ test). Data are reported as mean \pm SEM. ${ }^{*} p<0.05 ;$; $\mathrm{s}$, not significant. Scale bars, $20 \mu \mathrm{m}$.

tion occurs only at the axonal terminal when growth cones split into two equal sized branches (Acebes and Ferrús, 2000). Collateral branches often form along main axons interstitially (Gallo, 2011; Kalil and Dent, 2014); however, they sometimes form near axonal terminals where they emerge as short protrusions that are behind the axonal growth cone and shorter than the axonal tip (Szebenyi et al., 1998; Davenport et al., 1999). In WT neurons, bifurcations occurred at a rate of $0.15 \pm 0.03 \mathrm{event} / \mathrm{h}$ and retracted at $0.05 \pm 0.02$ event $/ \mathrm{h}$, whereas collateral branches occurred at $0.20 \pm 0.04 \mathrm{event} / \mathrm{h}$ and retracted at $0.07 \pm 0.02$ event/h.

$M a p 7^{-1-}$ neurons were able to extend branches at a rate of $0.60 \pm 0.10 \mathrm{event} / \mathrm{h}$, a $71 \%$ increase compared with WT neurons (Fig. $2 H)$, with a $53 \%$ increase in bifurcation $(0.23 \pm 0.05$ event $/ \mathrm{h})$ and $85 \%$ increase in collateral branch formation $(0.37 \pm$ 0.08 event/h). However, the frequency of overall branch retraction was increased dramatically by $309 \%$ and reached $0.34 \pm 0.08$ total event/h in neurons lacking MAP7 (Fig. 2I), with a significant increase $(>270 \%)$ in both bifurcations $(0.14 \pm 0.04$ event/h) and collateral branches $(0.19 \pm 0.05$ event $/ \mathrm{h})$.

Furthermore, nascent branches in WT neurons reached $23.9 \pm 2.3 \mu \mathrm{m}$ in length before retraction $(29.6 \pm 5.0 \mu \mathrm{m}$ for bifurcation and $20.6 \pm 1.6 \mu \mathrm{m}$ for collateral) and had a lifetime of $141 \pm 18 \mathrm{~min}(189 \pm 39 \mathrm{~min}$ for bifurcation and $120 \pm 16 \mathrm{~min}$ for collateral; Fig. $2 \mathrm{~J}, K$ ). These two parameters showed no sig- nificant change in $\mathrm{Map7}^{-1-}$ neurons, as the length of all nascent branches reached $20.8 \pm 2.1 \mu \mathrm{m}$ (Fig. $2 J$; $23.3 \pm 4.2 \mu \mathrm{m}$ for bifurcations and $19.1 \pm 2.0 \mu \mathrm{m}$ for collateral) before retraction and their lifetime was $144 \pm 24 \mathrm{~min}$ (Fig. $2 \mathrm{~K}$; $162 \pm 46 \mathrm{~min}$ for bifurcations and $134 \pm 28 \mathrm{~min}$ for collateral). Together, the analysis of Map7 knock-out neurons suggests that MAP7 promotes axonal branch maturation mainly by reducing retraction of newly formed branches.

\section{MAP7 is both necessary and sufficient to generate stable} microtubules in DRG axons

To understand the mechanism of the MAP7 function in promoting branch maturation, we examined MAP7 regulation of microtubule stability in different axonal regions. Because microtubules are bundled together in neurons, we used a previously described nocodazole treatment method (Baas and Black, 1990) to obtain an approximation of the level of stable and dynamic microtubules. We first tested this method by dissociating E17 rat DRG neurons to high concentrations of nocodazole $(6.6 \mu \mathrm{M})$ for $15 \mathrm{~min}$ before methanol extraction and fixation. This method (denoted as NocF) removes dynamic microtubules and leaves only stable microtubules (Baas and Black, 1990; Fig. 3A). For comparison, we also fixed neurons without nocodazole treatment (CtrlF), which preserved both dynamic and stable microtubules in axons. In both cases, we measured the level of 


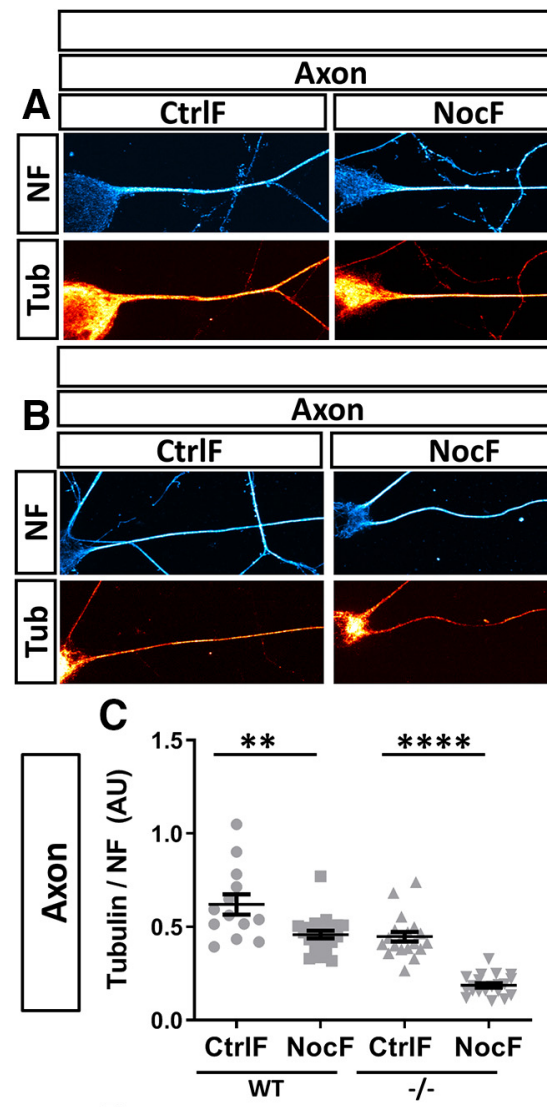

D

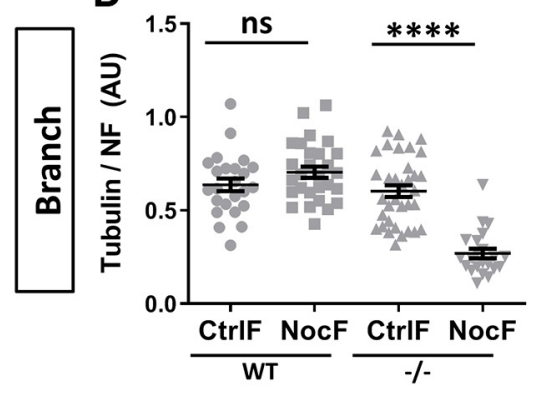

sistant to depolymerization, whereas the axonal shaft contains mixed populations of dynamic and stable microtubules, as previously shown (Song and Brady, 2015). This difference correlates well with endogenous MAP7, which is often localized at branch junctions (Tymanskyj et al., 2018) and occasionally in proximal axons (Fig. $3 D$ ). In the presence of MAP7, we found little change in the tubulin level between CtrlF and NocF (Fig. 3H). In contrast, regions with no or low levels of endogenous MAP7 showed a 13\% decrease in the microtubule level between CtrlF and NocF (Fig. 3I). Such correlation suggests a role for endogenous MAP7 in protecting microtubules from depolymerization and hence increasing stable microtubules in axons and especially at branch junctions.

To determine the requirement of MAP7 for preserving microtubule stability, we analyzed mouse $M a p 7^{-1-}$ neurons using the NocF method. As endogenous MAP7 afforded protection from depolymerization, we predicted that the absence of MAP7 would make neurons more susceptible to nocodazole-induced microtubule depolymerization. Consistent with the prediction, comparison of WT mouse neurons between CtrlF and NocF treatments shows a $28 \%$ decrease in the microtubule level in axons after NocF (Fig. $4 A, C)$ but the difference was greater in $M a p 7^{-1-}$ neurons, in which the microtubules level was reduced by $61 \%$ after NocF (Fig. $4 B, C$ ). For comparison, the NF level remained unaffected in CtrlF and NocFtreated neurons of either genotype (data not shown), similar to that in rat neurons. Moreover, analysis by CtrlF and NocF shows that branch junctions contain mostly stable microtubules in WT neurons (Fig. $4 A, B, D$ ) but lose $35 \%$ stable microtubules in neurons lacking MAP7 (Fig. 4D). These data thus support the role of MAP7 in maintaining stable microtubules in neurons.

To further support this role of MAP7 in axons, we analyzed stable microtubules in E17 rat DRG neurons overexpressing MAP7-EGFP, which closely mimicked the $\alpha$-tubulin (red; Fig. 3C) and normalized them to the level of NF, which remains unaffected by nocodazole treatments (Fig. $3 E$ ) and serves as an internal control to account for variations in axonal calibers. The difference between the normalized levels of stable microtubules after NocF and total microtubules after CtrlF, which represents the level of dynamic microtubules, was compared in two axonal regions (Fig. $3 B, C$ ). In the axon shaft, the level of microtubules after NocF was $\sim 10 \%$ lower than the total microtubules after CtrlF (Fig. $3 F$ ). However, at the branch junctions, the total microtubules had no significant difference between NocF and CtrlF (Fig. 3G). These results show that microtubules at mature branch junctions are mostly stable and re- localization of endogenous MAP7 and were enriched at branch junctions as previously shown (Tymanskyj et al., 2018). Overexpression increased the levels of MAP7 by $37 \%$ at branch junctions [160 \pm 8 a.u. (Ctrl) vs $219 \pm 19$ a.u. (MAP7), $p=0.0065, t$ test $]$ and $26 \%$ within limited axonal regions [116 \pm 0.3 a.u. (Ctrl) vs $147 \pm 11$ a.u. (MAP7), $p=0.0111, t$ test] based on MAP7-specific antibody staining. Consistent with the analysis above, nontransfected neurons showed a $26 \%$ decrease in microtubules after NocF only in axons but not in branch junctions (Fig. $4 E, F$ ). In contrast, overexpression of MAP7-EGFP within the axon was able to prevent the loss of microtubules after NocF (Fig. 4E). Furthermore, MAP7-EGFP did not alter the levels of microtu- 

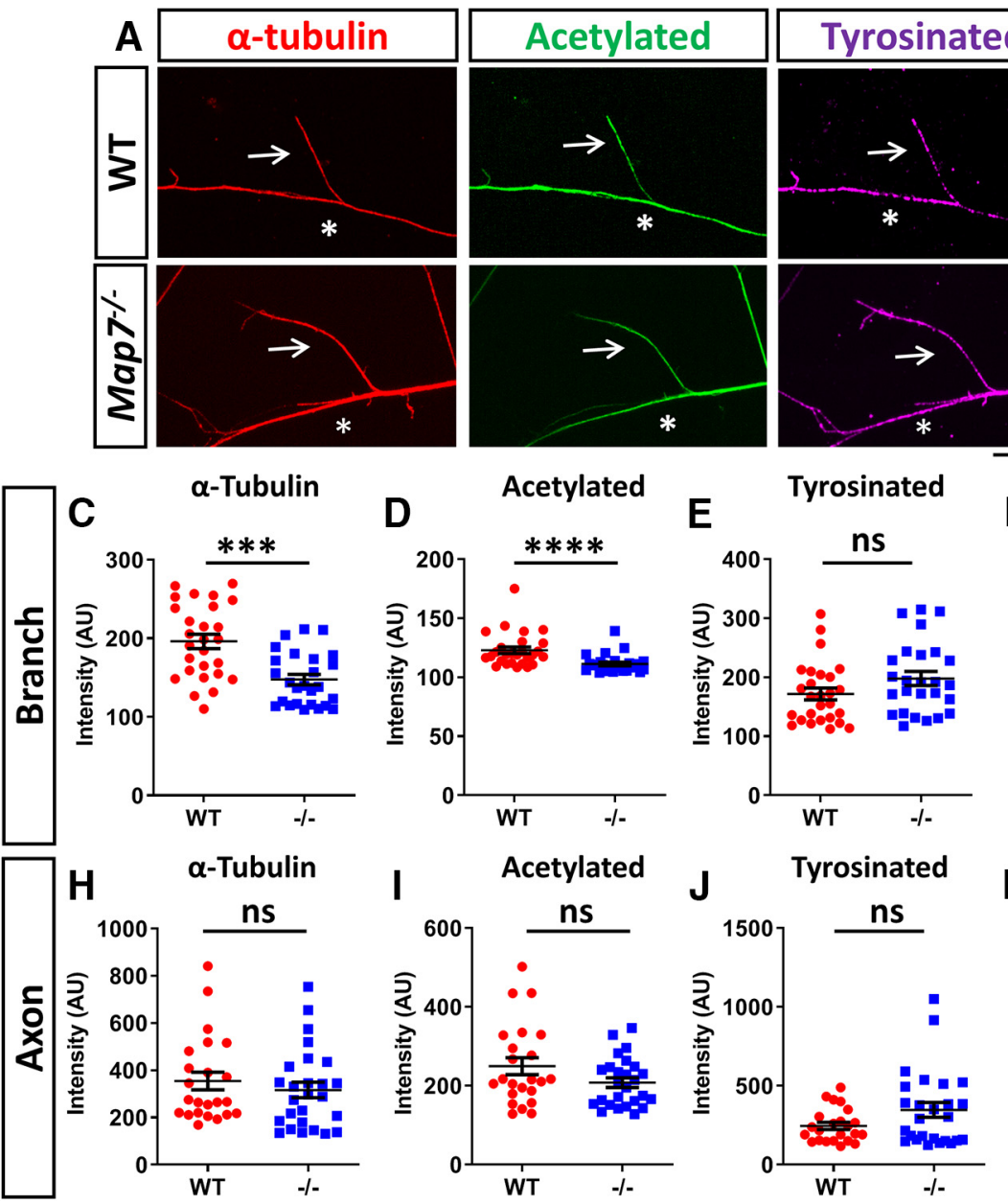

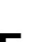

F
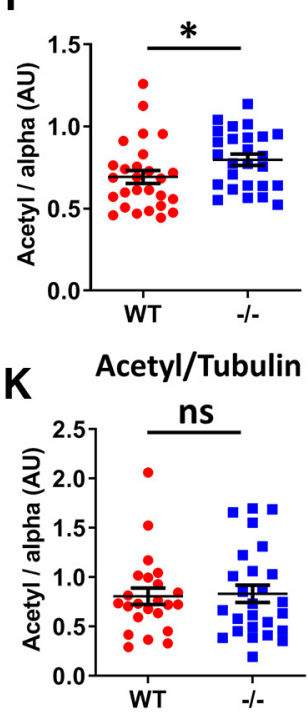
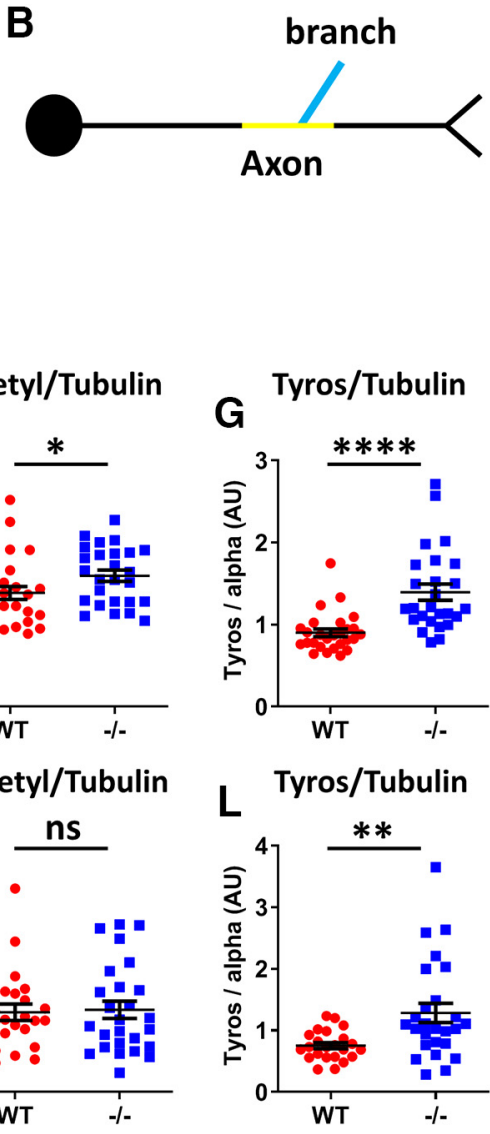

Figure 5. MAP7 regulates stable microtubules in nascent branches. $\boldsymbol{A}$, Fluorescence images of nascent branches (arrows) emerging from an axon (asterisks) labeled for $\alpha$-tubulin (red), acetylated tubulin (green), and tyrosinated tubulin (magenta) in WT and Map $7^{-1-}$ neurons. $\boldsymbol{B}$, Schematic of axonal regions analyzed: branch (blue) and axon (yellow). $\boldsymbol{C}-\boldsymbol{L}$, , Comparison of fluorescence signals in nascent branches $(\boldsymbol{C}-\boldsymbol{G})$ or axons $(\boldsymbol{H}-\boldsymbol{L})$ of WT and Map $7^{-I-}(-/-)$ neurons for total $\alpha$-tubulin $(\boldsymbol{C}, \boldsymbol{H})$, acetylated (acetyl)- tubulin $(\boldsymbol{D}, \boldsymbol{I})$ and tyrosinated (tyros)- tubulin $(\boldsymbol{E}, \boldsymbol{J})$, as well as their ratios $(\boldsymbol{F}, \mathbf{G}, \boldsymbol{K}, \boldsymbol{L})$. Data are reported as mean \pm SEM. Mann-Whitney tests: $\boldsymbol{C}, p=0.0002 ; \boldsymbol{D}, p \leq 0.0001 ; \boldsymbol{E}, p=0.0894 ; \boldsymbol{F}, p=0.0181 ; \boldsymbol{G}, p<0.0001 ; \boldsymbol{H}, p=0.4092 ; \boldsymbol{I}, p=0.2519 ; \boldsymbol{J}, p=0.2519 ;$ $K, p=0.9763 ; L, p=0.0034$; for branches, $n=27$ (WT) or $n=26(-/-)$; for axons, $n=23$ (WT) and $n=26(-/-)$. ${ }^{*} p<0.05 ;{ }^{* *} p<0.01 ;{ }^{* * *} p<0.001$; ${ }^{* * * *} p<0.0001 ;$ ns, not significant. Scale bar, $20 \mu \mathrm{m}$.

bules between CtrlF and NocF at branch junctions, consistent with the endogenous MAP7 localization (Fig. $4 F$ ). Interestingly, the total tubulin levels normalized to NF increased in both axons (by 32\%) and branch junctions (by 50\%) in MAP7-expressing neurons when compared with control cells, suggesting a longterm effect of increased MAP7 expression on stable microtubules (Fig. 4E,F). For comparison, tau-EGFP, which was suggested to bind dynamic microtubules in axons (Qiang et al., 2018), behaved the same way as MAP7-EGFP, increasing stable and total microtubules in both axons and branch junctions (Fig. $4 E, F$ ). Thus, these experiments provide strong evidence that MAP7 is both necessary and sufficient to preserve microtubules against depolymerization by increasing microtubule stability in neurons.

Loss of MAP7 reduces total and stable microtubules but increases dynamic microtubules in nascent branches

As MAP7 was able to increase stable microtubules in localized regions of axons, we next asked how this function contributes to branch formation by analyzing stable and dynamic microtubules in nascent branches (defined as those between 5 and $20 \mu \mathrm{m}$ ), which can potentially grow into mature branch. Because NocF induces retraction of nascent branches, we examined microtu- bule stability by immunostaining of $\alpha$-tubulin for total microtubules, acetylated tubulin, a marker for long-lived and likely stable microtubules (Webster and Borisy, 1989; Garnham and RollMecak, 2012), and tyrosinated tubulin, which is associated with newly assembled and dynamic ends of microtubules (Webster et al., 1987; Ahmad et al., 1993; Fig. 5A,B).

We analyzed and compared these markers between WT and $M a p 7^{-1-}$ mouse neurons. Based on the $\alpha$-tubulin level, we found that $M a p 7^{-1-}$ neurons had a decreased level of total microtubules within the nascent branch (24\% decrease; Fig. $5 C$ ). We also found a $9 \%$ decrease in the level of acetylated tubulin (Fig. 5D), but interestingly, the acetylation density is increased by $19 \%$ as shown by the ratio of acetylated- and $\alpha$-tubulin (Fig. $5 F$ ), likely because of the compensation of other MAPs (see Discussion). For comparison, the levels of $\alpha$-tubulin and acetylated tubulin as well as the acetylation density in the axon directly below the branch showed no difference between WT and $\mathrm{Map7}^{-1-}$ axons (Fig. $5 H, I, K)$, suggesting that the decreases seen are specific to nascent branches, likely those attempting to transition from initiation to maturation. Finally, the total level of tyrosinated-tubulin, based on staining with a specific antibody (YL1/2; Baas and Black, 1990), showed no significant difference between WT and 
$M a p 7^{-1-}$ neurons in both branches and axonal regions (Fig. $5 E, J)$ but the relative level normalized to total $\alpha$-tubulin has a significantly increase (42\%) in $M a p 7^{-1-}$ neurons (Fig. 5G,L), indicating an increase in the proportion of dynamic microtubules when MAP7 is lost. Together, these results suggest that MAP7 influences the level of total and stable microtubules as well as dynamic microtubules in nascent branches.

\section{MAP7 binds the stable region of microtubules and generates an anti-depolymerization boundary}

To understand the spatial relationship between MAP7 and acetylated or tyrosinated microtubules, we analyzed MAP7 localization in COS cells, in which individual microtubules can be readily resolved. In nontransfected or EGFP-transfected control cells, we found that $78 \pm 14 \%$ of individual microtubules were composed of regions of acetylated tubulin, often localized more centrally, and regions of tyrosinated tubulin, which were present on the entire length of microtubules but enriched at the growing end (Fig. 6A,B), similar to the distribution described previously in neurons (Baas and Black, 1990; Brown et al., 1993). Interestingly, in MAP7-EGFP-expressing COS cells labeled for $\alpha$-tubulin, MAP7-EGFP was not present along the entire length of microtubules and was absent from the distal end as shown by a line scan across the length of microtubules (Fig. 6C,D). Colabeling revealed that the MAP7 signal colocalizes precisely with the acetylated region of microtubules and avoids the distal highly tyrosinated region, as shown by $46 \pm 13 \%$ of microtubules in transfected cells (Fig. $6 E-H$ ). These results suggest that MAP7 is highly associated with the acetylated region but absent from the dynamic region of microtubules.

To further demonstrate this spatial relationship of MAP7 with tyrosinated and acetylated or dynamic and stable regions of microtubules, we cotransfected MAP7 with the plus-end marker EB3-mCherry, which allowed us to follow the dynamic microtubule ends. EB3-labeled growing ends appeared as comets (Akhmanova and Steinmetz, 2010) that could be seen emerging from the tip of the MAP7-bound region of microtubules (Fig. $7 A)$. The EB3 comets then moved away, reflecting microtubule growth, but the MAP7 signal remained stationary, creating a gap between the two labels (Fig. 7A). This gap, representing the MAP7-free end, can be illustrated by the separation of two signals in the kymograph (Fig. 7B), supporting the idea that MAP7 does not bind to the dynamic region of microtubules.

Such a gap is dependent on full-length (FL) MAP7, as the amino-terminal microtubule binding domain $(\mathrm{N})$ (Tymanskyj et al., 2018) decorated the entire length of microtubules and followed the EB3-labeled microtubule end, leaving no gap between both signals (Fig. $7 A, B$ ). It is also unique to MAP7-FL, as tauEGFP behaved the same way as N-EGFP, showing no microtubule binding preference. This can be demonstrated by the measurement of the number of microtubules containing the gap (Fig. $7 C$ ). In the presence of MAP7-FL, nearly $57 \pm 2.8 \%$ of EB3-containig microtubules contain a gap whereas no gap was found in microtubules bound by MAP7-N or tau (Fig. 7C). Thus, these results reveal a unique property of MAP7 that binds to the stable region of microtubules and is absent from the dynamic growing region of the plus end.

To understand the functional role of microtubule-bound MAP7 in microtubule dynamics, we followed microtubule behaviors for a period of 2.4 min by kymographs (Fig. 7D) that covered multiple phases of dynamic instability. Such behavior can be visualized by the emergence, movement, and disappearance of EB3 comets, which represent rescue, growth, and catastrophe/disassembly of microtubules, respectively (Stepanova et al., 2003). We found that as shown above, the appearance of EB3 comets started from the MAP7-bound microtubule end and moved away from this boundary to create a gap in between. Interestingly, after its disappearance (Fig. 7D, asterisk), EB3 reappeared at the end of the MAP7-bound region that remained stationary. This behavior suggests that MAP7 creates a boundary between stable and dynamic regions of microtubules, where it prevents depolymerization and rescues polymerization. As a comparison, EB3 comets appear at the end of microtubules bound by N-EGFP or tau-EGFP, which always followed EB3 comets (Fig. 7D). Unlike MAP7-FL-EGFP that was restricted to the same region over time, N-EGFP or tau-EGFP expanded along the growing microtubules, leaving no gap between EB3 and EGFP signals. After EB3 comets disappeared, the N-EGFP or tau-EGFP signal also retreated along with shrinking microtubules and had no obvious effect on preventing depolymerization. Thus, these control proteins closely followed growing and shrinking microtubules. Finally, MAP7-FL does not affect microtubule assembly, as comparisons of EB3 speed and growth length showed no significant difference between the three conditions (Fig. $7 E, F$ ). Because the dynamic region of microtubules can grow and shrink repeatedly with little or no shrinkage of MAP7-bound microtubules, our data indicate that MAP7 can serve as a unique microtubule stabilizer that stops depolymerization and rescues polymerization.

To further demonstrate the anti-depolymerization and rescue function, we examined microtubules in cells treated with nocodazole $(6.6 \mu \mathrm{M}$, for $10 \mathrm{~min}$; Fig. $8 \mathrm{~A})$. Co-staining revealed that the distal MAP7-free region disappeared, but the MAP7bound region was resistant to nocodazole, as illustrated by line scans along individual microtubules (Fig. $8 B$, arrow). Labeling for both acetylated- and tyrosinated-tubulin after nocodazole treatment shows the loss of tyrosinated-only/acetylation-free tips of microtubules and the colocalization of both signals (Fig. $8 C, D$, arrows). We further characterized this feature by live imaging of EB3 comet behaviors after a brief nocodazole treatment followed by washout. Before nocodazole addition, MAP7-bound microtubules were rarely associated with EB3 comets (Fig. 8E, Pre), often displaying a gap between EB3 and MAP7-bound microtubules, as shown in the previous section. Addition of nocodazole $(15 \mu \mathrm{M})$ for 5 min completely removed EB3 comets, but the majority of MAP7-bound microtubules remained intact, again suggesting that MAP7-bound microtubules are stable and resist nocodazoleinduced depolymerization (Fig. 8E). For comparison, microtubules bound by N-EGFP or tau-EGFP often shrank after nocodazole treatment along with the disappearance of EB3 comets (Fig. $8 E$ ). Interestingly, after nocodazole washout, $89 \pm 6 \%$ EB3 comets reemerged from the tip of MAP7-bound microtubules (Fig. $8 E$, Washout). As a comparison, only $47 \pm 13 \%$ of EB3 comets (Fig. $8 F$ ) were associated with MAP7-bound microtubules at a given time before nocodazole treatment. This was in contrast to both N-EGFP and tau-EGFP, which were always associated with EB3 both before nocodazole treatment and after nocodazole washout (Fig. 8E,F). These data further demonstrate that MAP7-protected microtubules can rescue polymerization at the MAP7-bound boundary. Together, our data suggest that MAP7-bound microtubules reduce the extent of depolymerization and preserve microtubules for regrowth. 

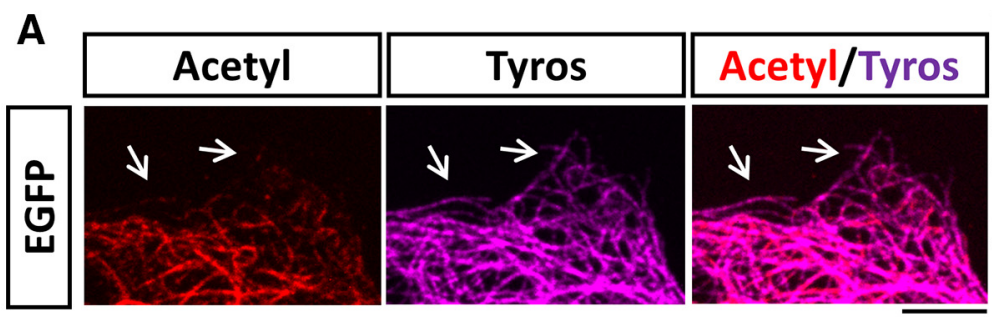

C
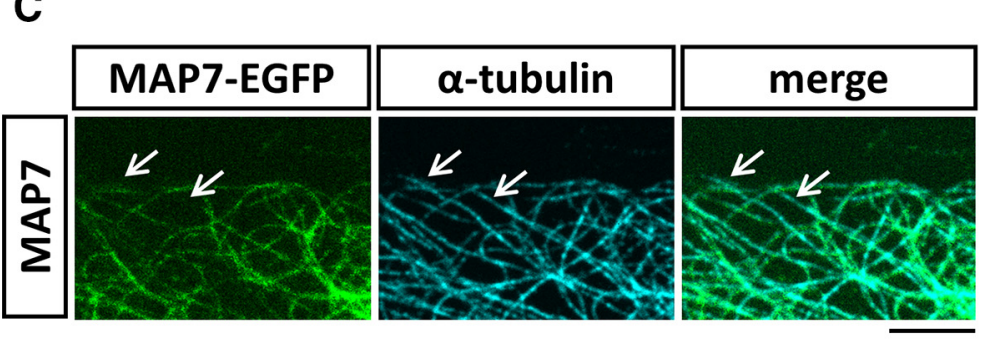
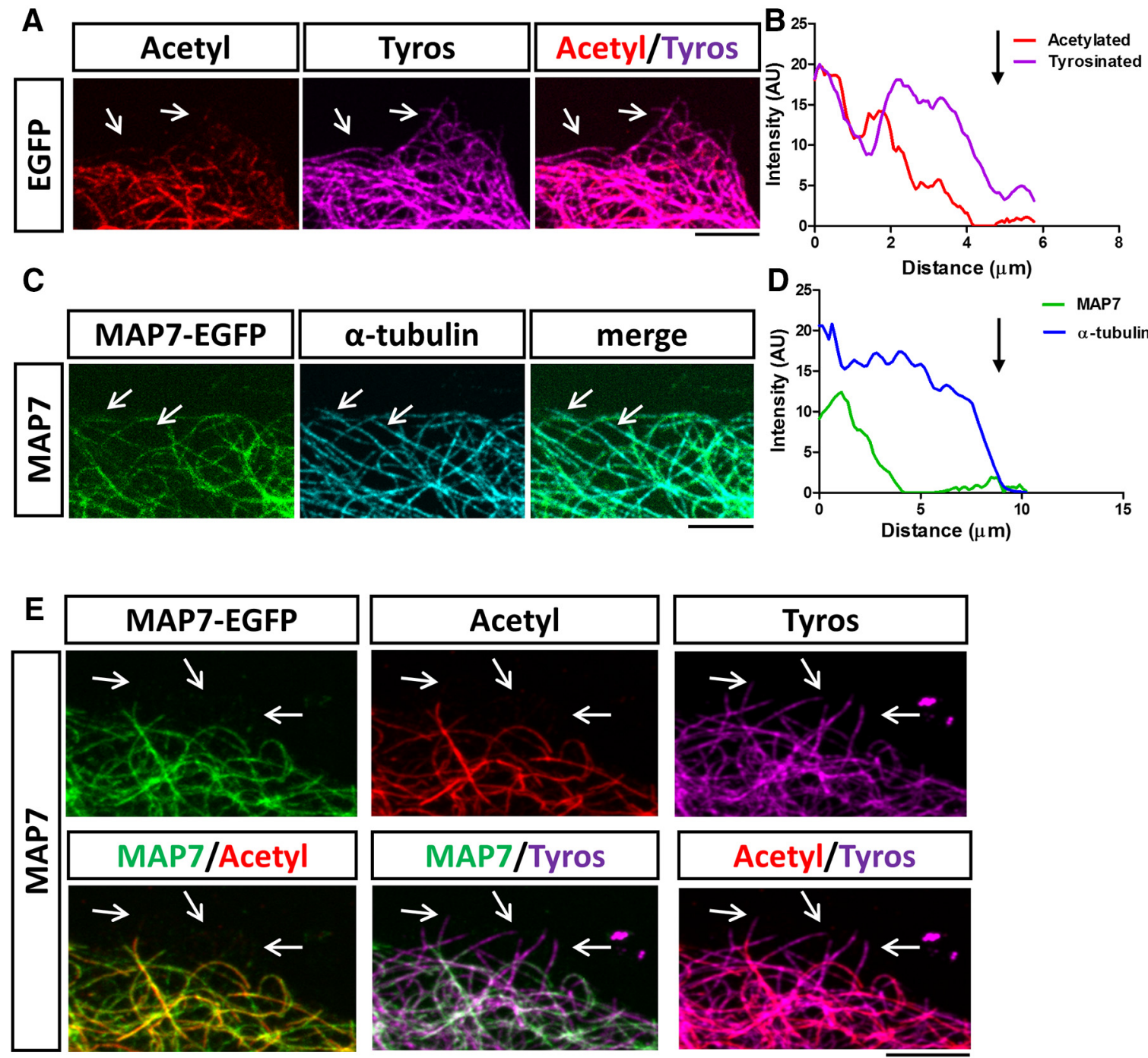

G

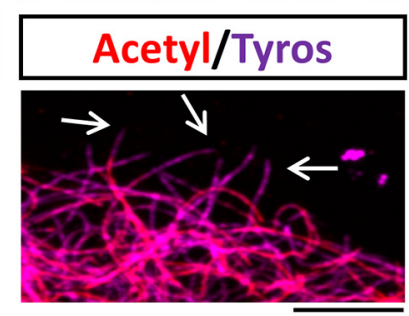

$\mathbf{F}$
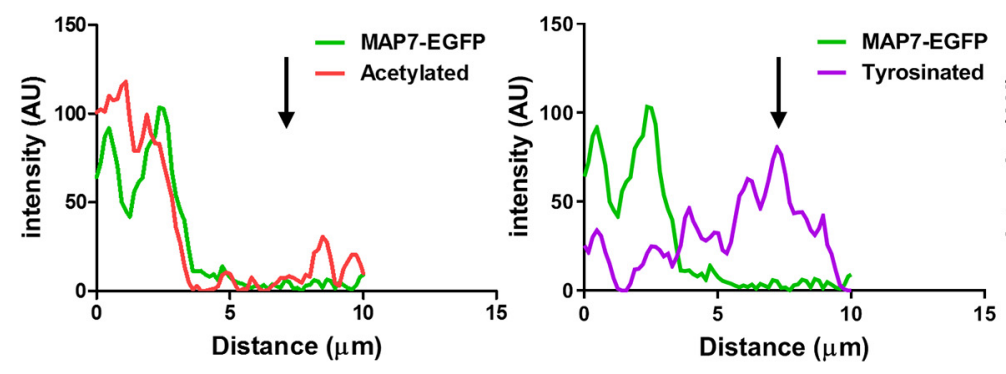

H

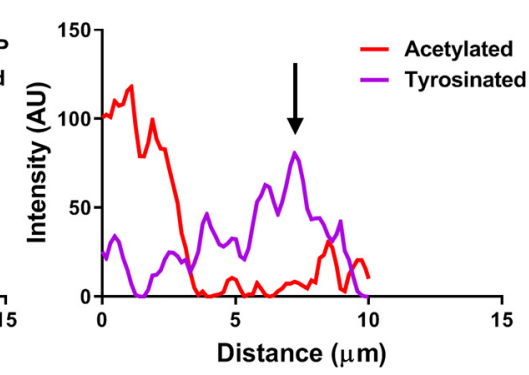

Figure 6. MAP7 binds to the acetylated region of microtubules. A, Fluorescence and merged images of a region of a control COS cell transfected with EGFP and labeled for acetylated (acetyl; red) and tyrosinated (tyros; magenta) microtubules (arrows). $\boldsymbol{B}$, Line scan along a single microtubule comparing acetylated and tyrosinated tubulin levels in $(\boldsymbol{A})$. $\boldsymbol{C}$, Fluorescence or merged images of a region of a $\operatorname{COS}$ cell expressing MAP7 (green) and labeled for $\alpha$-tubulin (cyan). Arrows identify microtubule regions lacking MAP7-EGFP. D, Line scans of MAP7 and $\alpha$-tubulin signals along a single microtubule from (C). E, Fluorescence (top rows) or merged (bottom rows) images of a region of a COScell transfected with MAP7-EGFP (green) and labeled for acetylated (acetyl; red) and tyrosinated (tyros; magenta) tubulin. Arrows point to regions of microtubules positive for tyrosinated tubulin but lacking both MAP7-EGFP and acetylated-tubulin signals. $\boldsymbol{F}-\boldsymbol{H}$, Line scans along a single microtubule comparing the spatial relationship of fluorescent signals between MAP7-EGFP and acetylated $(\boldsymbol{F})$ or tyrosinated tubulin $(\boldsymbol{G})$ or between acetylated and tyrosinated tubulin $(\boldsymbol{H})$. Scale bars: $5 \mu \mathrm{m}$.

MAP7 affects branch retraction caused by injury or nocodazole

The ability to stabilize microtubules can allow MAP7 to promote branch stability and prevent branch retraction during development. To further test this role, we asked whether MAP7 could prevent mature branch retraction after laser-induced severing
(Hammarlund et al., 2009; Yan et al., 2009). We cut a branch $30-40 \mu \mathrm{m}$ away from the branch junction in E17 rat DRG neurons expressing EGFP, and then followed the behavior of the injured branches by time-lapse imaging (Fig. 9A). After severing, EGFP-expressing control branches initially formed a retraction bulb (red arrows), and then rapidly retracted back to the main 

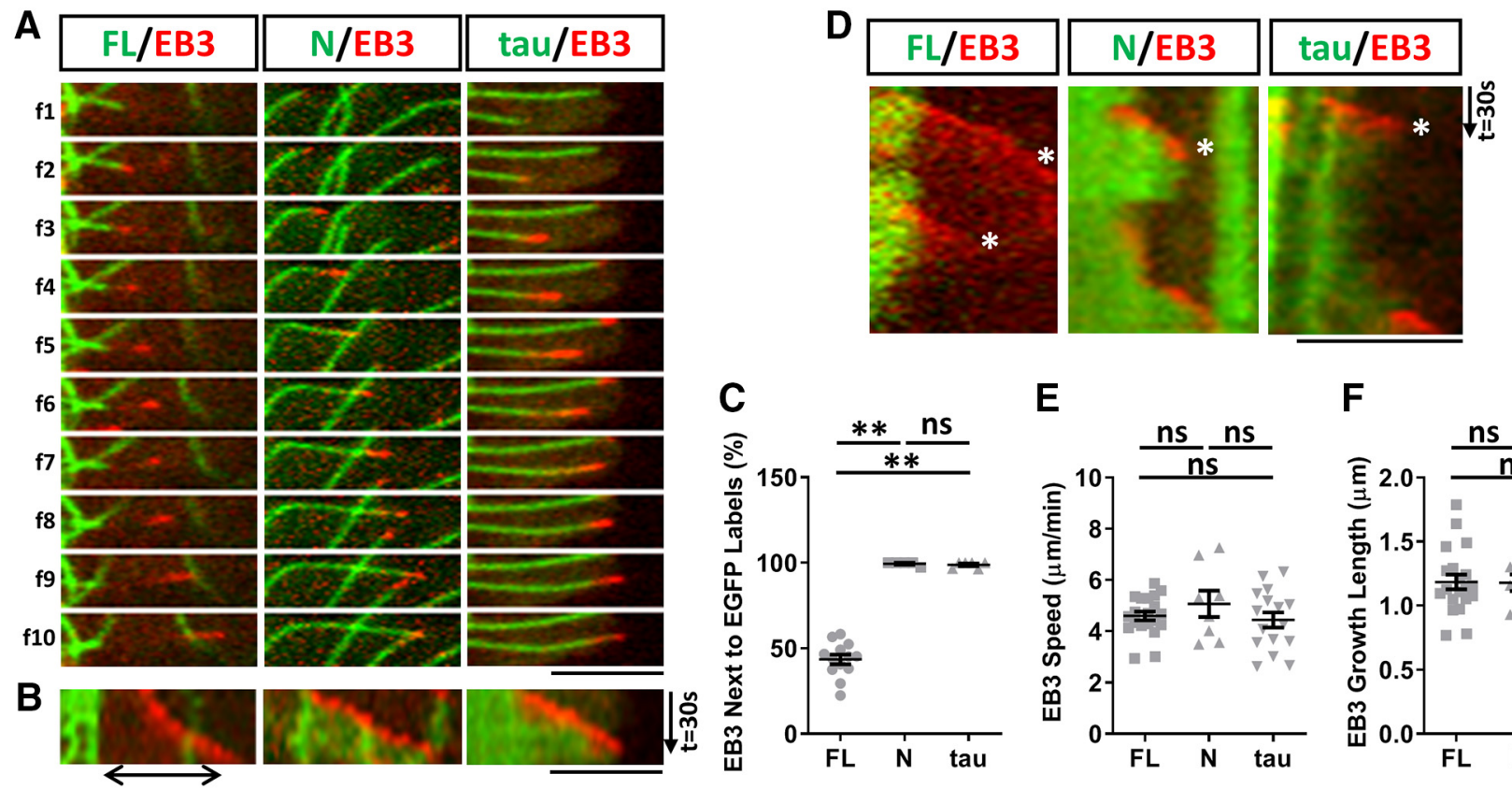

Figure 7. MAP7 creates a stable microtubule region that prevents depolymerization and rescues polymerization. $A$, Sequential fluorescence images (f1- $\mathrm{f10}$ ) of live COS cells expressing EB3-mCherry (red) with EGFP fusions (green) of MAP7-FL (FL), MAP7-N (N), or tau. Images were compressed at the Y-axis by 1.4X, and the time interval between images is 7.4s (FL) or 3.7s (N and tau). $\boldsymbol{B}$, Kymographs of EGFP and $m$ Cherry signals from $A$. A gap between FL-EGFP and EB3 is indicated by a line with double arrows. C, Quantification of the number of times EB3-mCherry comets are associated with MAP7-FL-EGFP, MAP7-N-EGFP, or tau-EGFP decorated microtubules (FL-N; $p=0.0011 ; F L-t a u ; p=0.0016, \mathrm{~N}$-tau; $p=0.9899, n=13$ for FL, $n=5$ for N and $n=6$ for tau, Kruskal-Wallis test). $\boldsymbol{D}$, Kymographs of EB3-mCherry (red) with EGFP fusions (green) of FL, $\mathrm{N}$ and tau showing growth and shrinkage of microtubules. Catastrophe events are marked by asterisks. $\boldsymbol{E}, \boldsymbol{F}$, Comparisons of EB3 speed ( $\boldsymbol{E} ; \mathrm{FL}-\mathrm{N}: p=0.5475 ; \mathrm{FL}$-tau: $p=0.8967 ; \mathrm{N}$-tau: $p=0.3692, n=20$ for $\mathrm{FL}, n=8$ for N, $n=16$ for tau, 0 ne-way ANOVA) and growth length $(\boldsymbol{F} ; \mathrm{FL}-\mathrm{N}: p=0.9983$; FL-tau: $p=0.6752 ; \mathrm{N}$-tau: $p=0.7314, n=20$ for FL, $n=8$ for N, $n=8$ for tau, one-way ANOVA) of polymerizing microtubules in transfected cells. Data are reported as mean \pm SEM. ${ }^{* *} p<0.01$; ns, not significant. Scale bars, $5 \mu \mathrm{m}$.

axon with a retraction rate of $0.82 \pm 0.11 \mu \mathrm{m} / \mathrm{min}$. We next examined the retraction response in neurons expressing MAP7EGFP and focused on branches with MAP7 concentrated at branch junctions. This revealed that expression of MAP7 greatly decreased the speed of retraction or die-back of the damaged branch to $0.17 \pm 0.04 \mu \mathrm{m} / \mathrm{min}$ (Fig. $9 B$ ). In many cases, the injured branch often showed little retraction after $80 \mathrm{~min}$ of imaging whereas the majority of EGFP neurons had undergone complete retraction by this point. As a comparison, DRG neurons expressing tau-EGFP, which was uniformly localized in the axons and branches, were able to reduce the retraction rate of injured axons $(0.49 \pm 0.14 \mu \mathrm{m} / \mathrm{min}$; Fig. $9 A-C)$, but not to the same extent as MAP7. These results support that the antidepolymerization and rescue functions of MAP7 may contribute to preventing axon retraction after injury.

To further test the effect of MAP7-mediated microtubule stability to branch retraction, we examined retraction induced by high doses of nocodazole that depolymerize microtubules. First, WT and $M a p 7^{-1-}$ neurons were treated with $15 \mu \mathrm{M}$ nocodazole, which was sufficient to induce retraction in the majority of axonal branches. Immediately after treatment, we recorded the retraction response from branch terminals by time-lapse imaging and plotted the retraction distance over time (Fig. 9D). In WT neurons, the branch retraction rate was $0.26 \pm 0.05 \mu \mathrm{m} / \mathrm{min}$ (Fig. $9 E)$; however, in mutant neurons, the rate was increased by 2.3 fold to $0.59 \pm 0.09 \mu \mathrm{m} / \mathrm{min}$, suggesting that microtubule stability exerted by MAP7 is important for preventing retraction. We next examined whether overexpression of MAP7 in rat DRG neurons could reduce the extent of nocodazole-induced retraction. Similar to that seen in the injury analysis, we found that the retraction rate in branches with MAP7 overexpression was decreased by twofold compared with EGFP (EGFP: $0.28 \pm 0.04 \mu \mathrm{m} / \mathrm{min}$; MAP7: $0.14 \pm 0.02 \mu \mathrm{m} / \mathrm{min}$; Fig. $9 F, G)$. As a control, overexpression of tau did not significantly reduce the retraction rate $(0.24 \pm 0.06 \mu \mathrm{m} / \mathrm{min})$. These data thus support the notion that MAP7 is both necessary and sufficient to prevent branch retraction by counteracting nocodazole-induced microtubule depolymerization.

\section{Discussion}

Axonal branch development requires reorganization of the cytoskeleton in nascent branches that undergo growth and retraction (Gallo, 2011). Here we demonstrate a key role for MAP7 in promoting branch maturation by reducing branch retraction. We show that MAP7 achieves this by stabilizing microtubules within branch junctions. We further demonstrate that MAP7 binding to microtubules creates a boundary and preserves a stable microtubule region to prevent depolymerization and rescue polymerization. This binding property provides a unique mechanism to regulate branch morphogenesis.

\section{MAP7 localized at branch junctions is required for branch} maturation by preventing branch retraction

Axon branching involves multiple steps, including initiation and maturation (Gallo, 2011; Gibson and Ma, 2011; Kalil and Dent, 2014). Previous cell biological analysis suggests that MAP7 is not involved in branch initiation, but rather promotes branch maturation (Tymanskyj et al., 2017, 2018). Our study of Map7 knockout neurons supports this function and reveals a new role of MAP7 in this step (Figs. 1,2). Consistent with overexpression studies (Tymanskyj et al., 2017), live cell imaging analysis revealed that the absence of MAP7 does not alter branch initiation 

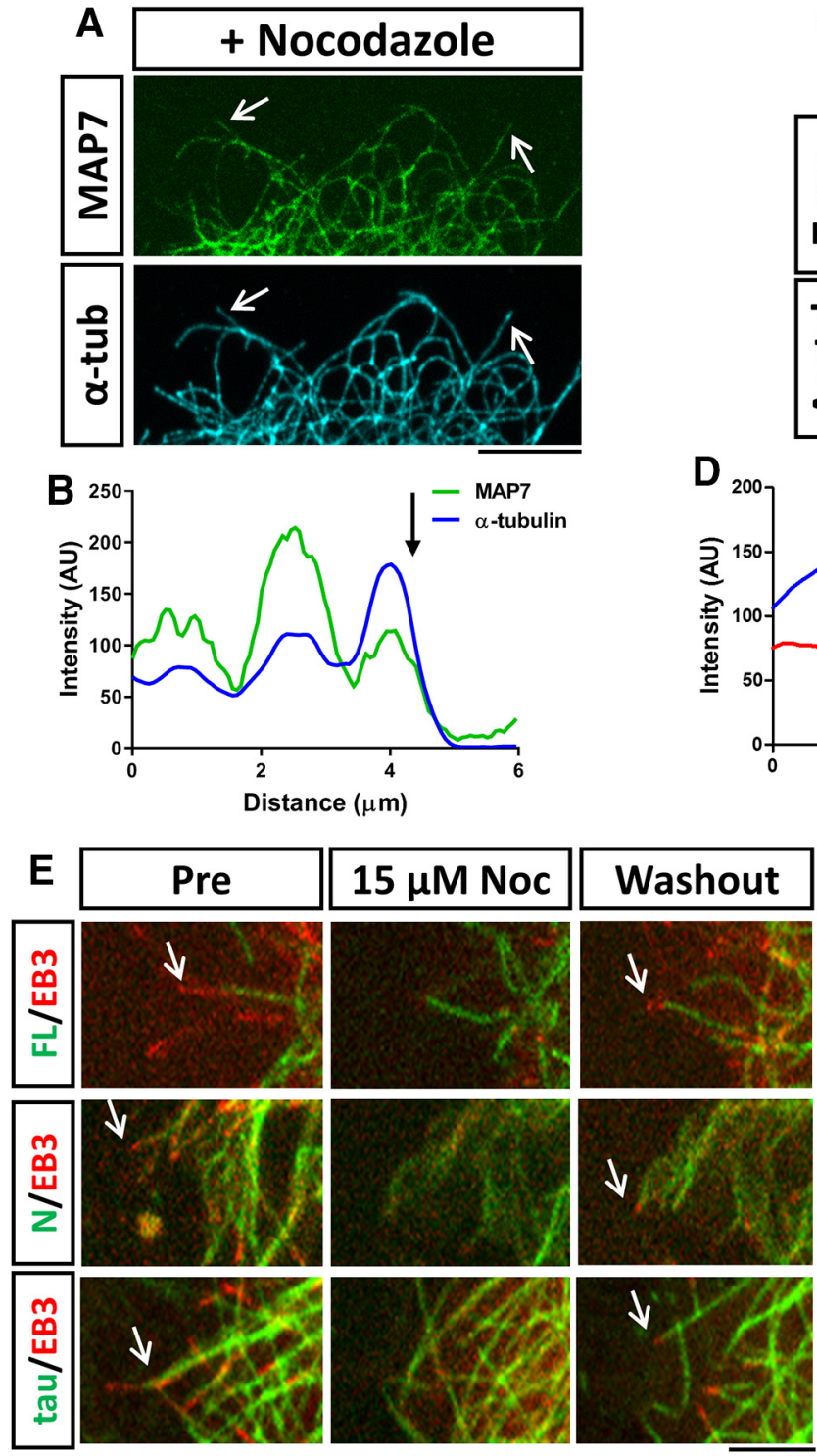
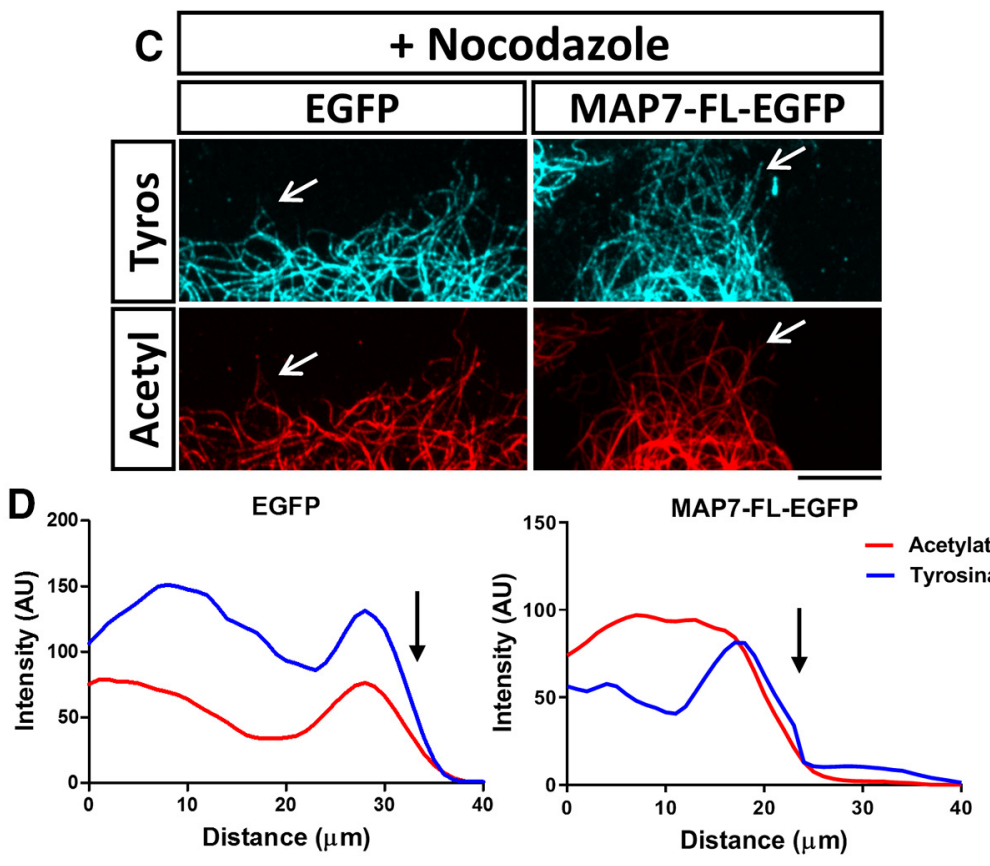

F

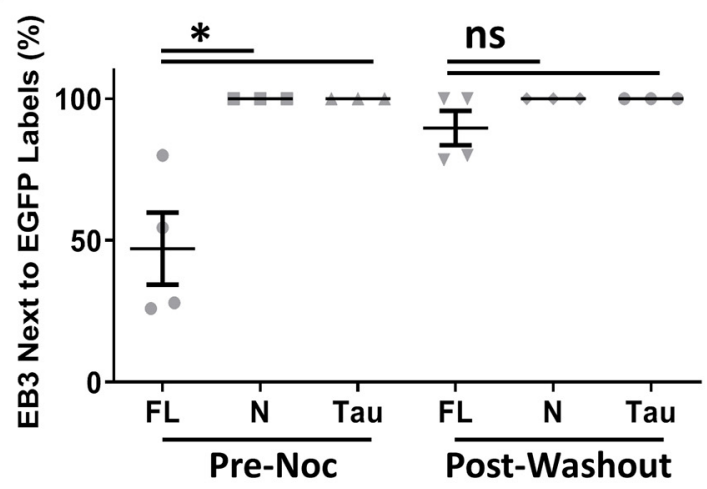

Figure 8. MAP7 prevents nocodazole-induced depolymerization and rescues polymerization after washout. $\boldsymbol{A}$, Fluorescence images of MAP7-EGFP and $\alpha$-tubulin staining (arrows) in a region of a COS cell transfected with MAP7-EGFP and treated with nocodazole $(6.6 \mu \mathrm{m})$. $\boldsymbol{B}$, Line scans along a single microtubule in $(\boldsymbol{A})$ reveal that the MAP7 signal terminates at the end (arrow) of the microtubule. C, Fluorescence images of a region of a COS cell transfected with EGFP or MAP7-EGFP and stained for $\alpha$-tubulin ( $\alpha$-tub) and acetylated-tubulin (actyl). $\boldsymbol{D}$, Line scans along single microtubules in ( $\boldsymbol{C}$ show that tyrosinated (tyros) microtubules colocalize with acetylated regions after nocodazole treatment. Arrows indicate the end of microtubules. $\boldsymbol{E}$, Snap shots of COS cell regions expressing MAP7-FL-EGFP, MAP7-N-EGP (green), or tau-EGFP along with EB3-mCherry (red), before (Pre) or during nocodazole treatment (Noc; $15 \mu \mathrm{m}$ ) or after nocodazole washout. Arrows point to EB3-containing growing microtubule ends. $F$, Quantification of FL, $\mathrm{N}$, and tau-bound microtubules associated with EB3 positive tips pre-nocodazole treatment and post-nocodazole washout (pre; N-FL; $p=0.0297 ; \mathrm{N}$-tau; $p \geq 0.999$, FL-tau; $p=0.0297 ;$ post; N-FL; $p=0.3685 ; \mathrm{N}$-tau; $p \geq 0.9999$, FL-tau; $p=0.3685, n=4$ for all constructs, Kruskal-Wallis test). Data are reported as mean \pm SEM. ${ }^{*} p<0.05$; ns, not significant. Scale bars, $5 \mu \mathrm{m}$.

but affects nascent branch maturation (Fig. 2). Interestingly, $M a p 7^{-l-}$ neurons produced an increase of branch growth but a much larger increase of branch retraction, leading to a net decrease in nascent branch maturation. Further analysis shows that such changes apply to different modes of branching (Fig. 2), suggesting a broader role MAP7 plays during branch morphogenesis. Although it is intriguing that MAP7 deletion also affects nascent branch growth, which could result from the increase of dynamic microtubules (see the next section), the larger effect on branch retraction in $M a p 7^{-1-}$ neurons reveals a critical role MAP7 plays during branch maturation. Importantly, this role is distinct from other MAPs, such as MAP1B, which has been implicated in branch initiation (Ketschek et al., 2015).
MAP7 promotes stable microtubules in mature and nascent branches

How does MAP7 prevent branch retraction? Here, we show that its ability to stabilize microtubules at the branch junctions is the key. Previous work has suggested that different axonal regions are composed of microtubules with different stability properties, with the distal region of the axon often being composed of more dynamic microtubules and the proximal region being more stable (Song and Brady, 2015). Here, we used a nocodazole fixation method (Baas et al., 1993) to show that microtubules present at mature branch junctions are highly stable compared with other regions of the axon (Fig. 3). We found that axonal regions with stable microtubules were correlated with high levels of endogenous MAP7; neurons cultured from Map7 knock-out mice had a 


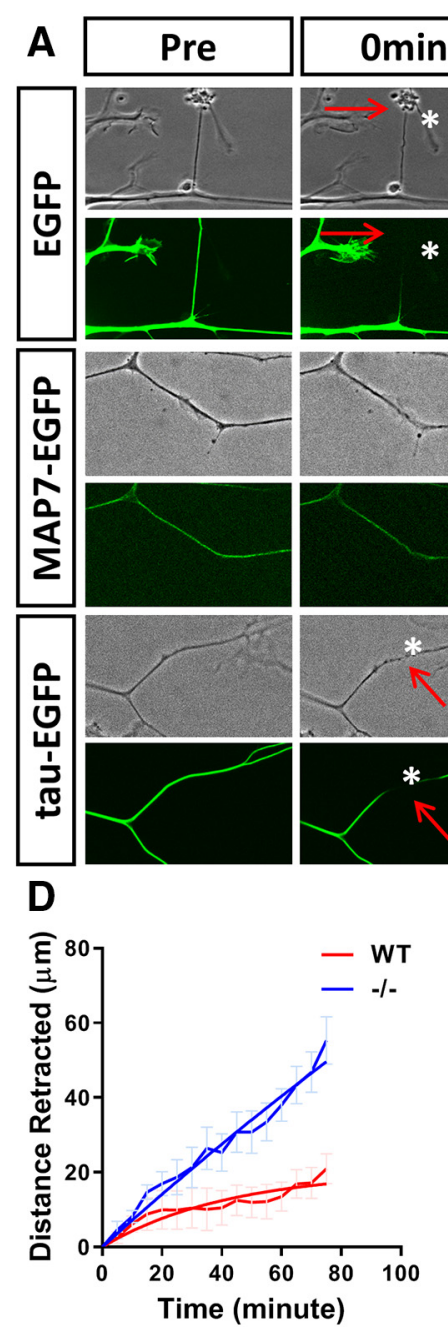

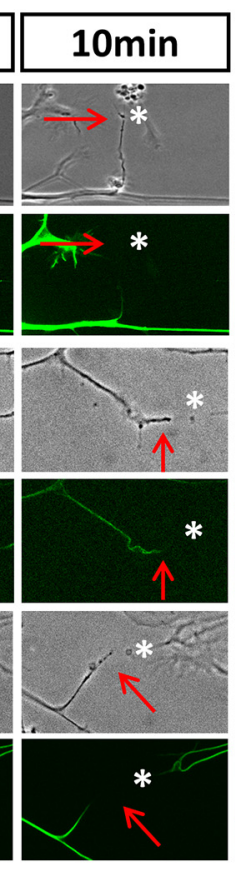

E

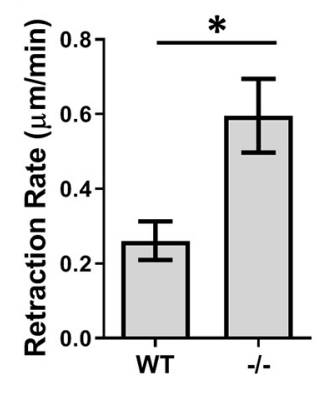

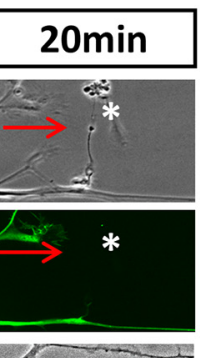

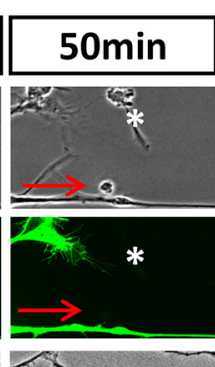

B

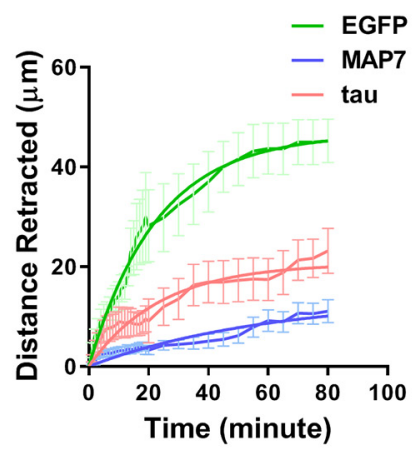

C

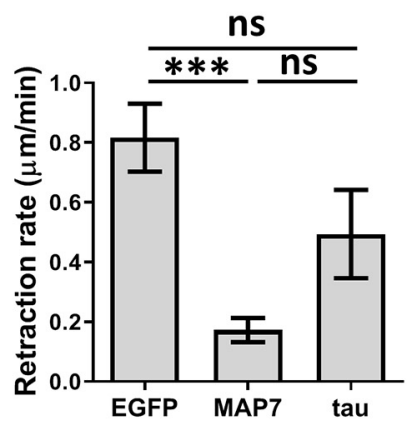

F

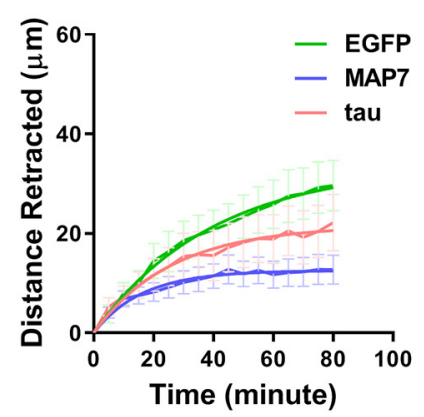

G

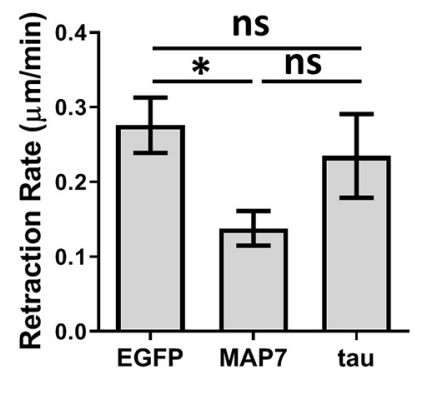

Figure 9. MAP7 prevents branch retraction caused by laser-induced severing or nocodazole-induced microtubule destabilization. $\boldsymbol{A}$, Phase contrast (top row) and EGFP (bottom row) images of DRG axons before (pre) and after laser-induced severing in neurons expressing EGFP, MAP7-EGFP, or tau-EGFP. The injured sites are marked by asterisks and the proximal branch terminals are marked by arrows. $\boldsymbol{B}$, The extent of retraction is plotted as distance retracted over time after severing. $\boldsymbol{C}$, Quantification and comparison of the axon retraction rate derived from $\boldsymbol{B}$ (EGFP-MAP7; $p=0.0002$, EGFP-tau; $p=0.0918 ;$ MAP7-tau; $p=0.0561 ; n=6$ for EGFP, $n=10$ for MAP7 and $n=6$ tau; one-way ANOVA). $\boldsymbol{D}, \boldsymbol{E}$, Time courses (D) and rates $(\boldsymbol{E})$ of retraction after nocodazole (15 $\mu$ m) addition to WT and Map7 ${ }^{-1-}(-1-)$ DRG neurons $(p=0.0222, n=6$ for WT and $n=9$ for $-1-, t$ test). $\boldsymbol{F}, \mathbf{G}$, Time courses $(\boldsymbol{F})$ and rates $(\boldsymbol{G})$ of retraction in rat DRG neurons overexpressing MAP7 or tau after nocodazole (15 $\mu \mathrm{M}$ ) addition (EGFP-MAP7; $p=0.0302$, EGFP-tau; $p=0.7490$; MAP7-tau; $p=0.2378, n=15$ for EGFP, $n=12$ for MAP7 and $n=9$ for tau, one-way ANOVA). Data are reported as mean \pm SEM. ns, not significant. ${ }^{*} p<0.05,{ }^{* * *} p<0.001$. Scale bar, $20 \mu \mathrm{m}$.

large decrease in the level of stable microtubules at branch junctions; and conversely overexpression of MAP7 was able to stablilize microtubules in axonal regions that are normally more dynamic (Figs. 3,4). These data strongly suggest that MAP7 is a microtubule stabilizer in axons, espeically at mature branch junctions. Furthermore, the increase in stable microtubules by tau overexpression suggests that high levels of tau have different effects from endogenous tau shown by the recent knockdown study (Qiang et al., 2018). It is worth noting that although MAP7 and tau overexpression has similar effects on microtubule stability, Map7 knock-out reduces stable microtubules but tau knockdown increases them (Qiang et al., 2018), an intriguing difference that may reflect their unique interactions with microtubules.

Our analysis of total microtubules as well as posttranslational modifications of microtubules further support this function of MAP7 during branch maturation. We found that MAP7 is required for maintaining the level of total and acetylated microtubules in nascent branches (Fig. 5). Acetylation of microtubules, often associated with long-lived and likely stable microtubules
(Portran et al., 2017), has been shown to increase the resilience and ensure persistence of microtubules (Xu et al., 2017). It is thus possible that these properties from acetylated microtubules are critical for branch stability, and without MAP7, stable microtubules are reduced in nascent branches, thereby increasing the chance of retraction.

Moreover, in nascent branches of $\mathrm{Map7}^{-1-}$ neurons, we found that the number of tyrosinated microtubules, which represent dynamic microtubules, did not change, suggesting an alteration of the balance between acetylated/tyrosinated microtubules. Thus, MAP7 may be also required to control the ratio of stable and dynamic microtubules during branch maturation. This interpretation is consistent with a recent study demonstrating that tau is responsible for preserving the labile domains of microtubules (Qiang et al., 2018) as well as another recent study showing that MAP7 is able to evict and replace tau from binding along microtubules (Monroy et al., 2018). As tau has a uniform distribution within axons (Dotti et al., 1987) and MAP7 is enriched at branch junctions, these 
proteins may coordinate to regulate microtubule stability. Once MAP7 enters nascent branches, it replaces tau bound to dynamic microtubules and makes them more stable, thereby creating stable microtubule arrays to prevent branch retraction. Thus, MAP7 is likely a key MAP that stabilizes microtubules during branch maturation.

\section{MAP7 is associated with the stable region of microtubules and serves as an anti-depolymerization factor}

Individual microtubules are composed of a stable region, located proximal to the minus end, and a dynamic/labile region at the plus end (Baas et al., 2016). Detailed analysis of MAP7 localization along individual microtubules revealed that MAP7 binding is restricted to specific regions, which are also acetylated and corresponded with the stable region of a microtubule. Interestingly, such a binding property creates a MAP7-free end that is highly tyrosinated and sensitive to nocodazole. We demonstrate such regional distinctions with EB3 labeling and found that MAP7 does not follow or associate with the dynamic plus ends. Further analysis of microtubule dynamics has revealed that the MAP7-bound microtubule region creates an anti-depolymerization boundary to rescue microtubule polymerization (Figs. 7,8 ). Although further investigation is needed to understand the underlying mechanism, this feature of MAP7 is likely responsible for stopping dynamic microtubules from complete disassembly and thereby preventing retraction of nascent branches. As MAP7 is able to decrease the extent of depolymerization, this will increase the life of microtubules and thus increase the likelihood of acetylation. As far as we are aware, this unique binding has not been observed for other MAPs. Indeed, tau, a known microtubule stabilizer, binds to the entire length of microtubules and does not create a similar tau-free gap or stop microtubule depolymerization. Thus, this unique microtubule binding property of MAP7, likely mediated by peptides outside the $\mathrm{N}$ domain, provides a novel mechanism to regulate microtubule stability in neurons. As recent work (Hooikaas et al., 2019; Pan et al., 2019) reported diverse functions of mammalian MAP7 homologs, it would thus be interesting to determine whether these homologs have similar microtubule binding properties as MAP7.

\section{MAP7 expressed at branch junctions reduces branch retraction after laser-induced severing}

In addition to promoting branch maturation, our study also demonstrates that the increased microtubule stability exerted by MAP7 plays a role in established branches (Fig. 9A-C). Laserinduced injury to mature branches produced rapid retraction or die-back of the injured branch back to the main axon (Hammarlund et al., 2009; Yan et al., 2009). However, expression of MAP7 was able to greatly decrease or inhibit retraction. Using a nocodazole retraction assay to examine the effects of microtubule depolymerization yielded similar results, as loss of MAP7 enhanced the branch retraction rate whereas MAP7 overexpression reduced it (Fig. 9D-G). This suggests that manipulation of MAP7 expression or activity could potentially reduce the retraction distance of an injured axonal branch and decrease the distance needed to regrow. As DRG axons often retract up to $300 \mu \mathrm{m}$ away from the site of injury (Kerschensteiner et al., 2005), reducing retraction distance will likely aid functional recovery of regenerating branches. The importance of stabilizing microtubules in regeneration has been demonstrated previously in injured adult axons, where the addition of the microtubule stabilizing drug Taxol promotes regeneration of axons in the spinal cord (Hellal et al., 2011; Sengottuvel et al., 2011; Bradke et al., 2012). Our study comparing MAP7 and tau shows that different microtubule stabilization mechanisms have different impacts on branch regeneration. Thus, manipulating MAP7 could potentially provide a new way to promote branch regeneration after nerve injury (Tuszynski and Steward, 2012).

In summary, we have demonstrated a function of MAP7 in preventing branch retraction by increasing microtubule stability at branch junctions. This function is based on its unique interaction with microtubules, which is likely conferred by two microtubule binding sites (Tymanskyj et al., 2018). In addition to preventing branch retraction, the ability of MAP7 to stabilize microtubules could also promote branch growth by directly recruiting the motor protein kinesin-1 (Bulinski and Bossler, 1994; Sung et al., 2008; Barlan et al., 2013; Monroy et al., 2018; Tymanskyj et al., 2018) and providing a stable microtubule track for kinesin-mediated transport (Nakata and Hirokawa, 2003; Jacobson et al., 2006; Konishi and Setou, 2009). Thus, MAP7 represents a new class of MAPs that play critical roles in branch morphogenesis during axonal development.

\section{References}

Acebes A, Ferrús A (2000) Cellular and molecular features of axon collaterals and dendrites. Trends Neurosci 23:557-565.

Ahmad FJ, Pienkowski TP, Baas PW (1993) Regional differences in microtubule dynamics in the axon. J Neurosci 13:856-866.

Akhmanova A, Steinmetz MO (2010) Microtubule + TIPs at a glance. J Cell Sci 123:3415-3419.

Applegate KT, Besson S, Matov A, Bagonis MH, Jaqaman K, Danuser G (2011) plusTipTracker: quantitative image analysis software for the measurement of microtubule dynamics. J Struct Biol 176:168-184.

Baas PW, Black MM (1990) Individual microtubules in the axon consist of domains that differ in both composition and stability. J Cell Biol 111:495509.

Baas PW, Ahmad FJ, Pienkowski TP, Brown A, Black MM (1993) Sites of microtubule stabilization for the axon. J Neurosci 13:2177-2185.

Baas PW, Rao AN, Matamoros AJ, Leo L (2016) Stability properties of neuronal microtubules. Cytoskeleton 73:442-460.

Barlan K, Lu W, Gelfand VI (2013) The microtubule-binding protein ensconsin is an essential cofactor of kinesin-1. Curr Biol 23:317-322.

Bilimoria PM, de la Torre-Ubieta L, Ikeuchi Y, Becker EB, Reiner O, Bonni A (2010) A JIP3-regulated GSK3beta/DCX signaling pathway restricts axon branching. J Neurosci 30:16766-16776.

Bradke F, Fawcett JW, Spira ME (2012) Assembly of a new growth cone after axotomy: the precursor to axon regeneration. Nat Rev Neurosci 13: 183-193.

Brill MS, Kleele T, Ruschkies L, Wang M, Marahori NA, Reuter MS, Hausrat TJ, Weigand E, Fisher M, Ahles A, Engelhardt S, Bishop DL, Kneussel M, Misgeld T (2016) Branch-specific microtubule destabilization mediates axon branch loss during neuromuscular synapse elimination. Neuron 92:845-856.

Brown A, Li Y, Slaughter T, Black MM (1993) Composite microtubules of the axon: quantitative analysis of tyrosinated and acetylated tubulin along individual axonal microtubules. J Cell Sci 104:339-352.

Bulinski JC, Bossler A (1994) Purification and characterization of ensconsin, a novel microtubule stabilizing protein. J Cell Sci 107:2839-2849.

Davenport RW, Thies E, Cohen ML (1999) Neuronal growth cone collapse triggers lateral extensions along trailing axons. Nat Neurosci 2:254-259.

Dotti CG, Banker GA, Binder LI (1987) The expression and distribution of the microtubule-associated proteins tau and microtubule-associated protein 2 in hippocampal neurons in the rat in situ and in cell culture. Neuroscience 23:121-130.

Gallo G (2011) The cytoskeletal and signaling mechanisms of axon collateral branching. Dev Neurobiol 71:201-220.

Garnham CP, Roll-Mecak A (2012) The chemical complexity of cellular microtubules: tubulin post-translational modification enzymes and their roles in tuning microtubule functions. Cytoskeleton 69:442-463.

Gibson DA, Ma L (2011) Developmental regulation of axon branching in the vertebrate nervous system. Development 138:183-195.

Hammarlund M, Nix P, Hauth L, Jorgensen EM, Bastiani M (2009) Axon 
regeneration requires a conserved MAP kinase pathway. Science 323:802-806.

Hand RA, Khalid S, Tam E, Kolodkin AL (2015) Axon dynamics during neocortical laminar innervation. Cell Rep 12:172-182.

Hellal F, Hurtado A, Ruschel J, Flynn KC, Laskowski CJ, Umlauf M, Kapitein LC, Strikis D, Lemmon V, Bixby J, Hoogenraad CC, Bradke F (2011) Microtubule stabilization reduces scarring and causes axon regeneration after spinal cord injury. Science 331:928-931.

Hooikaas PJ, Martin M, Mühlethaler T, Kuijntjes GJ, Peeters CAE, Katrukha EA, Ferrari L, Stucchi R, Verhagen DGF, van Riel WE, Grigoriev I, Altelaar AFM, Hoogenraad CC, Rüdiger SGD, Steinmetz MO, Kapitein LC, Akhmanova A (2019) MAP7 family proteins regulate kinesin-1 recruitment and activation. J Cell Biol 218:1298-1318.

Jacobson C, Schnapp B, Banker GA (2006) A change in the selective translocation of the kinesin-1 motor domain marks the initial specification of the axon. Neuron 49:797-804.

Kalil K, Dent EW (2014) Branch management: mechanisms of axon branching in the developing vertebrate CNS. Nat Rev Neurosci 15:7-18.

Kapitein LC, Hoogenraad CC (2015) Building the neuronal microtubule cytoskeleton. Neuron 87:492-506.

Kerschensteiner M, Schwab ME, Lichtman JW, Misgeld T (2005) In vivo imaging of axonal degeneration and regeneration in the injured spinal cord. Nat Med 11:572-577.

Ketschek A, Jones S, Spillane M, Korobova F, Svitkina T, Gallo G (2015) Nerve growth factor promotes reorganization of the axonal microtubule array at sites of axon collateral branching. Dev Neurobiol 75:1441-1461.

Koizumi H, Tanaka T, Gleeson JG (2006) Doublecortin-like kinase functions with doublecortin to mediate fiber tract decussation and neuronal migration. Neuron 49:55-66.

Komada M, McLean DJ, Griswold MD, Russell LD, Soriano P (2000) E-MAP-115, encoding a microtubule-associated protein, is a retinoic acid-inducible gene required for spermatogenesis. Genes Dev 14:13321342.

Konishi Y, Setou M (2009) Tubulin tyrosination navigates the kinesin-1 motor domain to axons. Nat Neurosci 12:559-567.

Lewis TL Jr, Courchet J, Polleux F (2013) Cell biology in neuroscience: cellular and molecular mechanisms underlying axon formation, growth, and branching. J Cell Biol 202:837-848.

Metzger T, Gache V, Xu M, Cadot B, Folker ES, Richardson BE, Gomes ER, Baylies MK (2012) MAP and kinesin-dependent nuclear positioning is required for skeletal muscle function. Nature 484:120-124.

Monroy BY, Sawyer DL, Ackermann BE, Borden MM, Tan TC, OriMcKenney KM (2018) Competition between microtubule-associated proteins directs motor transport. Nat Commun 9:1487.

Nakata T, Hirokawa N (2003) Microtubules provide directional cues for polarized axonal transport through interaction with kinesin motor head. J Cell Biol 162:1045-1055.

O'Leary DD, Bicknese AR, De Carlos JA, Heffner CD, Koester SE, Kutka LJ, Terashima T (1990) Target selection by cortical axons: alternative mechanisms to establish axonal connections in the developing brain. Cold Spring Harb Symp Quant Biol 55:453-468.

Pan X, Cao Y, Stucchi R, Hooikaas PJ, Portegies S, Will L, Martin M, Akhmanova A, Harterink M, Hoogenraad CC (2019) MAP7D2 localizes to the proximal axon and locally promotes kinesin-1-mediated cargo transport into the axon. Cell Rep 26:1988-1999.e6.

Portran D, Schaedel L, Xu Z, Théry M, Nachury MV (2017) Tubulin acetylation protects long-lived microtubules against mechanical ageing. Nat Cell Biol 19:391-398.

Qiang L, Yu W, Andreadis A, Luo M, Baas PW (2006) Tau protects microtubules in the axon from severing by katanin. J Neurosci 26:3120-3129.
Qiang L, Yu W, Liu M, Solowska JM, Baas PW (2010) Basic fibroblast growth factor elicits formation of interstitial axonal branches via enhanced severing of microtubules. Mol Biol Cell 21:334-344.

Qiang L, Sun X, Austin TO, Muralidharan H, Jean DC, Liu M, Yu W, Baas PW (2018) Tau does not stabilize axonal microtubules but rather enables them to have long labile domains. Curr Biol 28:2181-2189.e4.

Rockland KS (2019) Corticothalamic axon morphologies and network architecture. Eur J Neurosci 49:969-977.

Sengottuvel V, Leibinger M, Pfreimer M, Andreadaki A, Fischer D (2011) Taxol facilitates axon regeneration in the mature CNS. J Neurosci 31:2688-2699.

Song Y, Brady ST (2015) Post-translational modifications of tubulin: pathways to functional diversity of microtubules. Trends Cell Biol 25:125-136.

Stepanova T, Slemmer J, Hoogenraad CC, Lansbergen G, Dortland B, De Zeeuw CI, Grosveld F, van Cappellen G, Akhmanova A, Galjart N (2003) Visualization of microtubule growth in cultured neurons via the use of EB3-GFP (end-binding protein 3-green fluorescent protein). J Neurosci 23:2655-2664.

Sung HH, Telley IA, Papadaki P, Ephrussi A, Surrey T, Rørth P (2008) Drosophila ensconsin promotes productive recruitment of kinesin-1 to microtubules. Dev Cell 15:866-876.

Szebenyi G, Callaway JL, Dent EW, Kalil K (1998) Interstitial branches develop from active regions of the axon demarcated by the primary growth cone during pausing behaviors. J Neurosci 18:7930-7940.

Tint I, Jean D, Baas PW, Black MM (2009) Doublecortin associates with microtubules preferentially in regions of the axon displaying actin-rich protrusive structures. J Neurosci 29:10995-11010.

Tuszynski MH, Steward O (2012) Concepts and methods for the study of axonal regeneration in the CNS. Neuron 74:777-791.

Tymanskyj SR, Lin S, Gordon-Weeks PR (2010) Evolution of the spatial distribution of MAP1B phosphorylation sites in vertebrate neurons. J Anat 216:692-704

Tymanskyj SR, Scales TM, Gordon-Weeks PR (2012) MAP1B enhances microtubule assembly rates and axon extension rates in developing neurons. Mol Cell Neurosci 49:110-119.

Tymanskyj SR, Yang B, Falnikar A, Lepore AC, Ma L (2017) MAP7 regulates axon collateral branch development in dorsal root ganglion neurons. J Neurosci 37:1648-1661.

Tymanskyj SR, Yang BH, Verhey KJ, Ma L (2018) MAP7 regulates axon morphogenesis by recruiting kinesin-1 to microtubules and modulating organelle transport. Elife 7:e36374.

Webster DR, Borisy GG (1989) Microtubules are acetylated in domains that turn over slowly. J Cell Sci 92:57-65.

Webster DR, Gundersen GG, Bulinski JC, Borisy GG (1987) Differential turnover of tyrosinated and detyrosinated microtubules. Proc Natl Acad Sci U S A 84:9040-9044.

Xu Z, Schaedel L, Portran D, Aguilar A, Gaillard J, Marinkovich MP, Théry M, Nachury MV (2017) Microtubules acquire resistance from mechanical breakage through intralumenal acetylation. Science 356:328-332.

Yan D, Wu Z, Chisholm AD, Jin Y (2009) The DLK-1 kinase promotes mRNA stability and local translation in C. elegans synapses and axon regeneration. Cell 138:1005-1018.

Yu W, Qiang L, Solowska JM, Karabay A, Korulu S, Baas PW (2008) The microtubule-severing proteins spastin and katanin participate differently in the formation of axonal branches. Mol Biol Cell 19:1485-1498.

Zhao Z, Wang Z, Gu Y, Feil R, Hofmann F, Ma L (2009) Regulate axon branching by the cyclic GMP pathway via inhibition of glycogen synthase kinase 3 in dorsal root ganglion sensory neurons. J Neurosci 29:13501360 . 